\title{
Higgs-stoponium mixing near the stop-antistop threshold
}

\author{
Geoffrey T. Bodwin, ${ }^{1, *}$ Hee Sok Chung, ${ }^{1,2, \dagger}$ and Carlos E. M. Wagner ${ }^{1,3, \$}$ \\ ${ }^{1}$ High Energy Physics Division, Argonne National Laboratory, Argonne, Illinois 60439, USA \\ ${ }^{2}$ Theory Department, CERN, 1211 Geneva 23, Switzerland \\ ${ }^{3}$ Enrico Fermi Institute, University of Chicago, Chicago, Illinois 60637, USA
}

(Received 5 October 2016; published 20 January 2017)

\begin{abstract}
Supersymmetric extensions of the standard model contain additional heavy neutral Higgs bosons that are coupled to heavy scalar top quarks (stops). This system exhibits interesting field theoretic phenomena when the Higgs mass is close to the stop-antistop production threshold. Existing work in the literature has examined the digluon-to-diphoton cross section near threshold and has focused on enhancements in the cross section that might arise either from the perturbative contributions to the Higgs-to-digluon and Higgs-to-diphoton form factors or from mixing of the Higgs boson with stoponium states. Near threshold, enhancements in the relevant amplitudes that go as inverse powers of the stop-antistop relative velocity require resummations of perturbation theory and/or nonperturbative treatments. We present a complete formulation of threshold effects at leading order in the stop-antistop relative velocity in terms of nonrelativistic effective field theory. We give detailed numerical calculations for the case in which the stop-antistop Green's function is modeled with a Coulomb-Schrödinger Green's function. We find several general effects that do not appear in a purely perturbative treatment. Higgs-stop-antistop mixing effects displace physical masses from the threshold region, thereby rendering the perturbative threshold enhancements inoperative. In the case of large Higgsstop-antistop couplings, the displacement of a physical state above threshold substantially increases its width, owing to its decay width to a stop-antistop pair, and greatly reduces its contribution to the cross section.
\end{abstract}

DOI: 10.1103/PhysRevD.95.015013

\section{INTRODUCTION}

In extensions of the standard model (SM), new heavy particles typically appear. For example, supersymmetric extensions of the SM include heavy stop quarks (stops), which are the supersymmetric partners of a top quark [1-5]. A stop quark and a stop antiquark (antistop) can bind to form a spectrum of stoponium states. The decays of these states into two photons potentially provide a clean signal for their detection. However, stoponium states typically have rather small gluon-fusion cross sections times branching ratios into two photons [6-12].

Extensions of the SM can also contain heavy Higgs bosons [13]. The presence of new Higgs doublets is motivated by weak-scale extensions of the SM that aim to address the disparity between the electroweak and Planck scales and to provide explanations of the origins of flavor and of the matter-antimatter asymmetry. In these theories, the SM description is recovered in the so-called decoupling regime, in which the masses of the heavy Higgs bosons become large. In such a regime, the heavy neutral

\footnotetext{
*gtb@anl.gov

hee.sok.chung@cern.ch

"cwagner@anl.gov
}

Published by the American Physical Society under the terms of the Creative Commons Attribution 3.0 License. Further distribution of this work must maintain attribution to the author(s) and the published article's title, journal citation, and DOI.
Higgs bosons may decay into pairs of third-generation fermions, including top quarks. Owing to the presence of these tree-level decays, the branching ratio of loop-induced decay processes is suppressed, making it difficult to observe the decay of the heavy Higgs boson to two photons.

The interplay of a heavy stop-antistop system with a heavy Higgs boson whose mass is near the stop-antistop production threshold results in interesting and intricate new phenomena. Loop-induced processes may be enhanced in the presence of heavy quarks or squarks that are strongly coupled to the heavy Higgs boson. In supersymmetric extensions of the SM, the dimensionful coupling of stops to the heavy Higgs bosons is governed by the Higgsino mass parameter $\mu$. Loop-induced processes may be significantly modified if the heavy quarks or squarks have masses that are comparable to that of the heavy Higgs boson. The modification to loop-induced processes may be especially important if there is a production threshold for heavy particle-antiparticle pairs that is close to the Higgs mass [14].

Near threshold, QCD perturbation-expansion contributions of relative order $n$ may receive $1 / v^{n}$ enhancements, where $v$ is half of the relative velocity between the stop and the antistop in the stop-antistop center-of-momentum (CM) frame. Such enhanced contributions are typically proportional to $\alpha_{s}^{n}\left(m_{\tilde{t}} v\right) / v^{n}$, where $\alpha_{s}$ is the strong-interaction running coupling and $m_{\tilde{t}}$ is the stop-quark mass. The presence of these enhanced contributions requires a resummation of the perturbative contributions, which, among 
other things, takes into account the formation of stopantistop bound states.

The issue of gluon-fusion Higgs production and decay to diphotons at the stop-antistop production threshold has been addressed recently in Ref. [15]. The authors of Ref. [15] recognized the possibility that Higgs-stoponium mixing and the formation of stop-antistop bound states could have a significant effect on the $g g \rightarrow H \rightarrow \gamma \gamma$ rate. They pointed out, as is stressed in Ref. [16], that stoponium effects lead to Higgs-digluon $(\mathrm{Hgg})$ and Higgs-diphoton $(H \gamma \gamma)$ form factors that are enhanced relative to the form factors that are obtained in fixed-order perturbative calculations. We note, though, that the description in Refs. $[15,16]$ does not explicitly account for all of the Higgs-stoponium mixing effects [6].

In this article, we provide a detailed analysis of the interplay between a heavy Higgs boson and a heavy stopantistop pair for Higgs masses that are close to the stopantistop production threshold. ${ }^{1}$ We take into account threshold enhancements to the stop-antistop amplitudes by means of the analogues for scalar quarks of the effective field theories nonrelativistic QED (NRQED) [17] and nonrelativistic QCD (NRQCD) [18,19]. In this framework, we are also able to give a description of Higgs-stop-antistop mixing near threshold that incorporates fully the effects that are contained in the stop-antistop Green's function. ${ }^{2}$

In order to make contact with recent numerical work on the Higgs-stop-antistop system, we choose, in our numerical work, a stop mass of $375 \mathrm{GeV}$, and we assume that the Higgs mass is near the stop-antistop production threshold, which is $750 \mathrm{GeV}$. Much of the recent numerical work in the literature was motivated by initial results from the ATLAS and CMS experiments that showed excesses in rates for the process $p p \rightarrow \gamma \gamma$ at diphoton masses around $750 \mathrm{GeV}[20,21]$. (For an extensive list of theoretical work that is related to this signal, see Ref. [22].) However, we emphasize that our work is not tied to any particular phenomenological model and that it is aimed at understanding the general features of a Higgs-stop-antistop system near threshold.

\footnotetext{
${ }^{1}$ In the cases of SM extensions that contain more than one stop quark, we will assume that the heaviest stop mass eigenstate is significantly heavier than the lightest one. This implies that the Higgs mass is far below the threshold for production of a heaviest stop and a heaviest antistop. Therefore, the heaviest stop contribution becomes subdominant with respect to the lighteststop contribution. We will consider the heaviest stop contribution to be a perturbation to the rates that are computed in this work. It should be taken into account in precision studies. For large values of the trilinear Higgs-stop-antistop coupling, the coupling of the heavy stop to the Higgs boson is of the same order as and opposite in sign to the coupling of the lightest stop to the Higgs boson. Hence, the contribution of the heavy stop to the diphoton rate may be non-negligible in the regime of strong Higgs-stopantistop coupling.

${ }^{2}$ In Ref. [14], the effects of mixing between the Higgs boson and discrete stoponium resonances were considered.
}

We find that effects of Higgs-stop-antistop mixing go beyond the modification of the $H g g$ and $H \gamma \gamma$ form factors and significantly change the diphoton production rate near the stop-antistop threshold with respect to the rate that would be obtained from the simple addition of the Higgs and stoponium contributions. For Higgs masses near threshold, we find that several mechanisms that arise from Higgs-stopantistop mixing suppress the diphoton rate relative to the rates that are obtained in perturbative calculations: (1) for small stop widths, mixing significantly increases the width of the narrowest physical state relative to the width of the unmixed stoponium state; (2) mixing shifts masses of physical states away from the region in which form-factor enhancements occur; (3) mixing shifts some physical-state masses above threshold, where, in the case of strong Higgsstop-antistop couplings, the states develop large decay widths into stop-antistop pairs; (4) for strong Higgs-stopantistop couplings, the mixing changes the heights and widths of the physical resonances that lie below threshold.

In some of our numerical work, we employ rather large values of the Higgs-stop-antistop coupling. In some specific models of the Higgs-stop-antistop system, such large values of the coupling could lead to the presence of colorsymmetry-breaking minima in the potential for the vacua [23-25]. Since, in our work, we are not focused on any specific model realization of the Higgs-stop-antistop system, we will not study these possible constraints. However, they would have to be taken into account in the construction of detailed models.

The remainder of this article is organized as follows. In Sec. II, we describe the effective-field-theory approach that we employ. We also give formulas for the $g g \rightarrow \gamma \gamma$ amplitude that account fully for the Higgs-stop-antistop mixing. Section III contains a discussion of a simplified model in which the stop-antistop states are replaced by a single Breit-Wigner resonance. That model exhibits a number of the features of the full theory. In Sec. IV, we present and discuss our numerical results for the $g g \rightarrow \gamma \gamma$ amplitude and cross section as a function of the Higgs mass, relative to the stop-antistop threshold. Section V contains our conclusions.

\section{EFFECTIVE-FIELD-THEORY APPROACH}

\section{A. NRQED/NRQCD analogues for scalar quarks}

We wish to compute the amplitude for $g g \rightarrow \gamma \gamma$ in the presence of a Higgs boson with mass $m_{H}$ that couples to a heavy stop quark and a heavy antistop quark, each of which have mass $m_{\tilde{t}}$. We are concerned with the situation in which $m_{H}$ is near $2 m_{\tilde{t}}$, the threshold for stop-antistop production. Because the amplitude is computed near threshold, there can be important effects from the binding or near binding of the stop-antistop pair that are not captured in fixed-order perturbation theory. It is convenient to take these effects into account by making use of the analogues for scalar 
quarks of the effective field theories NRQED [17] and NRQCD $[18,19] .^{3}$

We will carry out the effective-field-theory computation at the leading nontrivial order in the heavy stop velocity $v$ in the stop-antistop CM frame, where $v$ is given by

$$
v=|\boldsymbol{p}| / m_{\tilde{t}}=\sqrt{2 m_{\tilde{t}} E} / m_{\tilde{t}} .
$$

Here, $\boldsymbol{p}$ is the 3 -momentum of the stop in the stop-antistop (or $\gamma \gamma$ ) CM frame,

$$
E=\sqrt{\hat{s}}-2 m_{\tilde{t}}
$$

is the nonrelativistic $\mathrm{CM}$ energy of the stop-antistop system, $\sqrt{\hat{s}}$ is the partonic CM energy,

$$
\sqrt{\hat{s}}=m_{\gamma \gamma}
$$

and $m_{\gamma \gamma}$ is the $\gamma \gamma$ mass.

The effective field theory is an expansion in powers of $v$. Hence, our calculation should be valid as long as $v$ is much less than 1. We expect corrections to our calculation of the $g g \rightarrow \gamma \gamma$ amplitude to be of relative order $v^{2}$.

The heavy stop part of the effective Lagrangian density that we will use, which is valid at the leading order in $v$, is

$$
\begin{aligned}
\mathcal{L}_{\tilde{t} \tilde{t}}= & \psi^{\dagger}\left(2 i m_{\tilde{t}} D_{0}+D^{2}\right) \psi+\chi^{\dagger}\left(2 i m_{\tilde{t}} D_{0}+D^{2}\right) \chi-i C_{H \tilde{t} \tilde{t}} H\left(\psi^{\dagger} \chi+\chi^{\dagger} \psi\right) \\
& +(i / 2) C_{g g \tilde{t} \tilde{t}} \frac{1}{N_{c}^{2}-1}\left(\psi^{\dagger} \chi+\chi^{\dagger} \psi\right) G_{\mu \nu}^{a} G^{a \mu \nu}+(i / 2) C_{\gamma \gamma \tilde{t} \tilde{t}}\left(\psi^{\dagger} \chi+\chi^{\dagger} \psi\right) F_{\mu \nu} F^{\mu \nu} \\
& +(i / 2) C_{g g H} \frac{1}{N_{c}^{2}-1} H G_{\mu \nu}^{a} G^{a \mu \nu}+(i / 2) C_{\gamma \gamma H} H F_{\mu \nu} F^{\mu \nu} \\
& -i C_{\tilde{t} \tilde{t} H \tilde{t} \tilde{t}} \frac{1}{N_{c}} \psi^{\dagger} \chi \chi^{\dagger} \psi+i \operatorname{Im} T_{\tilde{t} \tilde{t} \rightarrow g g \rightarrow \tilde{t} \tilde{t}} \frac{1}{N_{c}} \psi^{\dagger} \chi \chi^{\dagger} \psi,
\end{aligned}
$$

where $m_{\tilde{t}}$ is the stop pole mass, $\psi$ is the field that annihilates a stop, $\chi$ is the field that creates an antistop, $G_{\mu}^{a}$ is the gluon field with adjoint color index $a, G_{\mu \nu}^{a}$ is the gluon field strength with adjoint color index $a, A_{\mu}$ is the electromagnetic field, and $F_{\mu \nu}$ is the electromagnetic field strength. The covariant derivative contains both the electromagnetic field and the gluon field:

$$
D_{\mu}=\partial_{\mu}-i e e_{\tilde{t}} A_{\mu}-i g G_{\mu}^{a} t_{a},
$$

where $e$ is the electromagnetic coupling, $e_{\tilde{t}}$ is the stopquark charge, $g$ is the strong-interaction coupling, and $t_{a}$ is an $S U(3)$ matrix in the adjoint representation that is normalized to

$$
\operatorname{Tr} t_{a} t_{b}=T_{R} \delta_{a b}=(1 / 2) \delta_{a b} .
$$

In the calculations in this paper, we ignore the couplings of stops and antistops to the electromagnetic field, except in the annihilation of a stop-antistop pair into two photons. The $C_{i}$ 's are short-distance coefficients, which will be determined by matching the effective theory with full QED and full QCD at the stop-antistop threshold. The shortdistance coefficient $C_{\tilde{t} \tilde{t} H \tilde{t} \tilde{t}}$ takes into account the $t$-channel exchange of the heavy Higgs boson between the stop and

\footnotetext{
${ }^{3}$ In Ref. [26], an effective field theory for stoponium systems was developed, and a resummation of threshold logarithms in the stoponium production cross section was carried out by making use of soft-collinear effective theory [27]. However, the formalism in Ref. [26] does not address the possibility of stoponiumHiggs mixing.
}

the antistop. The quantity $\operatorname{Im} T_{\tilde{t} \tilde{t} \rightarrow g g \rightarrow \tilde{t} \tilde{t}}$ is also a shortdistance coefficient that accounts for decays of a stopantistop pair into two gluons. It is given by $(-2)$ times the imaginary part of the contribution to the stop-antistop forward $T$-matrix that contains a two-gluon intermediate state, evaluated at the stop-antistop threshold. Note that, because we are working at leading order in $v$, there are only $S$-wave couplings to the stop-antistop pairs.

\section{B. Computation of the short-distance coefficients}

In this section, we compute the short-distance coefficients in Eq. (4) by matching the effective theory to the full theory. Because our focus is on the formulation of the calculation and on the qualitative features of the threshold physics, we work at the lowest nontrivial order in the electromagnetic and strong couplings. Therefore, one should take care in comparing our numerical results with those in the literature, which often are performed at next-to-leading order, and, therefore, include two-loop effects in the couplings of the Higgs boson to digluons and diphotons [28-31]. ${ }^{4}$

We compute the short-distance coefficients that appear at the Born level by evaluating the corresponding amplitude in the full theory at the stop-antistop threshold. We compute

\footnotetext{
${ }^{4} \mathrm{An}$ expansion of in powers of $\alpha_{s}$ is valid for the short-distance coefficients, since they contain no $1 / v$ enhancements. However, in the computation of the effective-field-theory amplitudes in Sec. II C, the expansion in powers of $\alpha_{s}$ can fail because there are contributions to the stop-antistop Green's function in order $\alpha_{s}^{n}$ that are enhanced by factors $1 / v^{n}$. We compute these contributions to all orders in $\alpha_{s}$.
} 
the short-distance coefficients that appear at one-loop level by evaluating the one-loop amplitude in the full theory at threshold and subtracting the corresponding one-loop amplitude in the effective theory.

The short-distance coefficients $C_{g g \tilde{t} \tilde{t}}, C_{\gamma \gamma \tilde{t} \tilde{t}}$, and $C_{\tilde{t} \tilde{t} H \tilde{t} \tilde{t}}$ are easily obtained by carrying out Born-level calculations in full QCD at the stop antistop threshold. They are given by

$$
\begin{aligned}
C_{g g \tilde{t} \tilde{t}} & =8 i \pi \alpha_{s}\left(m_{\tilde{t}}\right) \frac{T_{R}}{\sqrt{N_{c}}}, \\
C_{\gamma \gamma \tilde{t} \tilde{t}} & =8 i \pi \alpha e_{\tilde{t}}^{2} \sqrt{N_{c}}, \\
C_{\tilde{t} \tilde{t} H \tilde{t} \tilde{t}} & =i N_{c} \frac{g_{H \tilde{t} \tilde{t}}^{2}}{4 m_{\tilde{t}}^{2}},
\end{aligned}
$$

where $\alpha=e^{2} /(4 \pi), e_{\tilde{t}}$ is the stop electromagnetic charge, $\alpha_{s}=g^{2} /(4 \pi)$, and $N_{c}=3$ is the number of $S U(3)$ colors. We have chosen the effective-field-theory factorization scale to be $m_{\tilde{t}}$, which accounts for the argument of $\alpha_{s}$. We have also taken the color-singlet projection of the stopantistop pairs, making use of the projector

$$
P_{i j}=\delta_{i j} / \sqrt{N_{c}}
$$

where $i$ and $j$ are the squark and antiquark color indices, respectively.

The short-distance coefficient $C_{H \tilde{t} \tilde{t}}$ is simply the Bornlevel Higgs-stop-antistop coupling, $g_{H \tilde{t} \tilde{t}}$, rescaled by a factor of $\sqrt{N_{c}}$ :

$$
C_{H \tilde{t} \tilde{t}}=i \sqrt{N_{c}} g_{H \tilde{t} \tilde{t}} \equiv-i \sqrt{N_{c}} \kappa m_{\tilde{t}}
$$

where we have normalized the Higgs coupling to stops in terms of the stop mass, with $\kappa$ being an adjustable parameter.

The short-distance coefficients $C_{g g H}$ and $C_{H \gamma \gamma}$ are generated by quark loops and stop loops. We take into account the $b$-quark, $t$-quark, and stop loops, which give the most important contributions. In full QCD, we have the amplitude [32-34]

$$
i \mathcal{M}_{g g \rightarrow H}(\hat{s})=\left(\epsilon_{1} \cdot \epsilon_{2}-\frac{\epsilon_{1} \cdot k_{2} \epsilon_{2} \cdot k_{1}}{k_{1} \cdot k_{2}}\right) \delta_{a b} \mathcal{A}_{g g \rightarrow H}(\hat{s}),
$$

where

$$
\begin{gathered}
\mathcal{A}_{g g \rightarrow H}(\hat{s})=\frac{i \alpha_{s}}{8 \pi} T_{F} \hat{s}\left[\frac{2}{2}\right. \\
A_{1 / 2}(\tau)=2[\tau+\tau(1-\tau) f(\tau)], \\
A_{0}(\tau)=-\tau[1-\tau f(\tau)], \\
f(\tau)= \begin{cases}\arcsin ^{2}(1 / \sqrt{\tau}) \\
-\frac{1}{4}\left[\log \left(\frac{1+\sqrt{1-\tau}}{1-\sqrt{1-\tau}}\right)-i \pi\right]^{2} & \tau<1,\end{cases}
\end{gathered}
$$

and

$$
\begin{gathered}
\tau_{b}=\frac{4 m_{b}^{2}}{\hat{s}}, \\
\tau_{t}=\frac{4 m_{t}^{2}}{\hat{s}}, \\
\tau_{\tilde{t}}=\frac{4 m_{\tilde{t}}^{2}}{\hat{s}} .
\end{gathered}
$$

Here, $\left(k_{1}, \epsilon_{1}\right)$ and $\left(k_{2}, \epsilon_{2}\right)$ are the (momentum, polarization) of the initial gluons, $a$ and $b$ are the gluon color indices, $m_{b}$ and $e_{b}$ are the bottom-quark mass and electric charge, $m_{t}$ and $e_{t}$ are the top-quark mass and electric charge,

$$
\begin{gathered}
g_{H b b}=\frac{g_{\mathrm{EW}} m_{b}}{2 m_{W}} \tan \beta, \\
g_{H t t}=-\frac{g_{\mathrm{EW}} m_{t}}{2 m_{W} \tan \beta},
\end{gathered}
$$

$$
g_{H \tilde{t} \tilde{t}}=-\kappa m_{\tilde{t}},
$$

$g_{\mathrm{EW}}$ is the electroweak coupling, $m_{W}$ is the $W$-boson mass, $\tan \beta$ is the ratio of heavy and light Higgs vacuum expectation values in a supersymmetric model, and we have listed the values of the couplings in the heavy-Higgs-boson decoupling limit, ignoring small deviations of the couplings from those values.

Similarly, in the case of $H \rightarrow \gamma \gamma$, we have the amplitude [34-37] 


$$
i \mathcal{M}_{H \rightarrow \gamma \gamma}(\hat{s})=\left(\epsilon_{3} \cdot \epsilon_{4}-\frac{\epsilon_{3} \cdot k_{4} \epsilon_{4} \cdot k_{3}}{k_{3} \cdot k_{4}}\right) \mathcal{A}_{H \rightarrow \gamma \gamma}(\hat{s}),
$$

where

$$
\mathcal{A}_{H \rightarrow \gamma \gamma}(\hat{s})=\frac{i \alpha}{8 \pi} N_{c} \hat{s}\left[\frac{2 g_{H b b}}{m_{b}} e_{b}^{2} A_{1 / 2}\left(\tau_{b}\right)+\frac{2 g_{H t t}}{m_{t}} e_{\tilde{t}}^{2} A_{1 / 2}\left(\tau_{t}\right)+\frac{g_{H \tilde{t} \tilde{t}}}{m_{\tilde{t}}^{2}} e_{t}^{2} A_{0}(\tau)\right],
$$

and $\left(k_{3}, \epsilon_{3}\right)$ and $\left(k_{4}, \epsilon_{4}\right)$ are the (momentum, polarization) of the final photons.

The corresponding quantities in the effective theory are produced by the stop loop that is generated by the $C_{H \tilde{t} \tilde{t}}$, $C_{g g \tilde{t} \tilde{t}}$, and $C_{\gamma \gamma \tilde{t} \tilde{t}}$ terms in the effective action. In the modified-minimal-subtraction $(\overline{\mathrm{MS}})$ scheme, we obtain

$$
\begin{aligned}
& \mathcal{A}_{g g \rightarrow H}^{\mathrm{eff}}=C_{g g \tilde{t} \tilde{t}} C_{H \tilde{t} \tilde{t}} \frac{-i}{16 \pi m_{\tilde{t}}} \sqrt{-m_{\tilde{t}}(E+i \epsilon)}, \\
& \mathcal{A}_{H \rightarrow \gamma \gamma}^{\mathrm{eff}}=C_{\gamma \gamma \tilde{t} \tilde{t}} C_{H \tilde{t} \tilde{t}} \frac{-i}{16 \pi m_{\tilde{t}}} \sqrt{-m_{\tilde{t}}(E+i \epsilon)} .
\end{aligned}
$$

The effective-theory amplitudes in Eq. (17) vanish at the stop-antistop threshold $(E=0)$. Therefore, the shortdistance coefficients $C_{g g H}$ and $C_{\gamma \gamma H}$ are obtained simply by evaluating $\mathcal{A}_{g g \rightarrow H}$ and $\mathcal{A}_{H \rightarrow \gamma \gamma}$ at stop-antistop threshold:

$$
C_{g g H}=\mathcal{A}_{g g \rightarrow H}\left(4 m_{\tilde{t}}^{2}\right),
$$

$$
C_{\gamma \gamma H}=\mathcal{A}_{H \rightarrow \gamma \gamma}\left(4 m_{\tilde{t}}^{2}\right)
$$

Finally, as we have mentioned, the short-distance coefficient $\operatorname{Im} T_{\tilde{t} \tilde{t} \rightarrow g g \rightarrow \tilde{t} \tilde{t}}$ is obtained by computing the contribution from a two-gluon intermediate state to the imaginary part of the stop-antistop forward $T$-matrix, evaluated at the stop-antistop threshold. By making use of unitarity, one can obtain this quantity simply from a cut diagram. The result is

$$
2 \operatorname{Im} T_{\tilde{t} \tilde{t} \rightarrow g g \rightarrow \tilde{t} \tilde{t}}=\frac{1}{\pi}\left|C_{g g \tilde{t} \tilde{t}}\right|^{2} .
$$

\section{Computation of the $g g \rightarrow \gamma \gamma$ amplitude}

In the nonrelativistic effective theory, the stop-antistop interactions can be taken into account by considering the stop-antistop Green's function

$$
G_{\tilde{t} \tilde{t}}(\hat{s})=P_{i j} P_{k l} \int d x_{0} e^{i\left(\sqrt{\hat{s}}-2 m_{\tilde{i}}\right) x_{0}}\left\langle 0\left|\chi_{i}^{\dagger}\left(x_{0}, \mathbf{0}\right) \psi_{j}\left(x_{0}, \mathbf{0}\right) \psi_{k}^{\dagger}(0, \mathbf{0}) \chi_{l}(0, \mathbf{0})\right| 0\right\rangle .
$$

Note that the fields in the Green's function are evaluated at zero spatial separation and that color-singlet projections of the initial stop and antistop and the final stop and antistop have been taken by making use of the projectors $P_{i j} P_{k l}$ [Eq. (7)]. The Green's function contains all of the effects of the $1 / v^{n}$ enhancements that we have mentioned.

The Green's function $G_{\tilde{t} \tilde{t}}(\hat{s})$ can be evaluated in a systematic expansion in powers of $v$ by considering a reformulation of the nonrelativistic effective theory in terms of an effective theory that is the scalar-squark analogue of potential NRQCD [38]. In this effective theory, the Green's function can be evaluated by considering interactions of the stop and antistop through nonrelativistic potentials. (The potentials scale with definite powers of $v$.) The $1 / v^{n}$ enhancements arise from $n$ exchanges of heavy-stop-antistop potentials. A resummation of the potential exchanges, through the use of the Schrödinger equation, brings the $1 / v^{n}$ enhancements under control. The resummation of the potential exchanges takes into account, among other things, the formation of stop-antistop bound states. The potentials incorporate both perturbative and nonperturbative effects. If $m_{\tilde{t}} v$ is sufficiently large, they are well approximated by perturbative expressions, but they are also valid when $m_{\tilde{t}} v$ is in the nonperturbative regime.

We can take into account the four-fermion terms in the effective action that are proportional to $C_{\tilde{t} \tilde{t} H \tilde{t} \tilde{t}}$ and $\operatorname{Im} T_{\tilde{t} \tilde{t} \rightarrow g g \rightarrow \tilde{t} \tilde{t}}$ by replacing $G_{\tilde{t} \tilde{t}}(\hat{s})$ with

$$
\tilde{G}_{\tilde{t} \tilde{t}}(\hat{s})=\frac{1}{G_{\tilde{t} \tilde{t}}^{-1}(\hat{s})(0,0, E)-C_{\tilde{t} \tilde{t} H \tilde{t} \tilde{t}}+\operatorname{Im} T_{\tilde{t} \tilde{t} \rightarrow g g \rightarrow \tilde{t} \tilde{t}}} .
$$

In the case of a stoponium state, this replacement accounts for the decay width into two gluons, which, for small values of the stop width, is the dominant stoponium decay width. ${ }^{5}$

\footnotetext{
${ }^{5}$ For large values of the coupling of the SM-like Higgs boson to a stop-antistop pair, the stoponium state can also decay with a significant rate into a pair of $125 \mathrm{GeV}$ Higgs bosons. This occurs, for instance, in the minimal supersymmetric standard model, for large values of the stop mixing parameters [39]. In our work, we have assumed that this coupling takes moderate values and, consequently, that the decay width of the stoponium state into pairs of SM-like Higgs bosons is much smaller than its decay width into gluon pairs.
} 
The coupling of the Higgs-boson to a stop-antistop pair leads to several contributions to the $g g \rightarrow \gamma \gamma$ amplitude. We write these contributions to the amplitude as

$$
\begin{aligned}
i \mathcal{M}(g g \rightarrow \gamma \gamma)_{i}= & \left(\epsilon_{1} \cdot \epsilon_{2}-\frac{\epsilon_{1} \cdot k_{2} \epsilon_{2} \cdot k_{1}}{k_{1} \cdot k_{2}}\right) \\
& \times\left(\epsilon_{3} \cdot \epsilon_{4}-\frac{\epsilon_{3} \cdot k_{4} \epsilon_{4} \cdot k_{3}}{k_{3} \cdot k_{4}}\right) \delta_{a b} A_{i}(g g \rightarrow \gamma \gamma) .
\end{aligned}
$$

There is a contribution in which the initial $g g$ pair transitions to the Higgs boson and the Higgs boson transitions to a $\gamma \gamma$ pair:

$$
\begin{aligned}
& A_{1}(g g \rightarrow \gamma \gamma) \\
& \quad=C_{g g H}\left[S_{H}(\hat{s})+S_{H}(\hat{s}) C_{H \tilde{t} \tilde{t}} \tilde{G}_{\tilde{t} \tilde{t}}(\hat{s}) C_{H \tilde{t} \tilde{t}} S_{H}(\hat{s})+\ldots\right] C_{\gamma \gamma H} \\
& \quad=C_{g g H} \frac{1}{S_{H}^{-1}(\hat{s})-C_{H \tilde{t} \tilde{t}}^{2} \tilde{G}_{\tilde{t} \tilde{t}}(\hat{s})} C_{\gamma \gamma H} \\
& =C_{g g H} \frac{i}{\hat{s}-m_{H}^{2}+i m_{H} \Gamma_{H}-i C_{H \tilde{t} \tilde{t}}^{2} \tilde{G}_{\tilde{t} \tilde{t}}(\hat{s})} C_{\gamma \gamma H} .
\end{aligned}
$$

Here,

$$
S_{H}=\frac{i}{\hat{s}-m_{H}^{2}+i m_{H} \Gamma_{H}}
$$

is the Higgs propagator (that is, the Higgs Green's function in the absence of Higgs-stop-antistop interactions), $m_{H}$ is the Higgs pole mass, and $\Gamma_{H}$ is the Higgs width.

There is also a contribution in which the initial $g g$ state transitions to a Higgs boson, which transitions to a stopantistop pair, which transitions to a $\gamma \gamma$ pair:

$$
\begin{aligned}
A_{2}(g g \rightarrow \gamma \gamma)= & C_{g g H} \frac{i}{\hat{s}-m_{H}^{2}+i m_{H} \Gamma_{H}-i C_{H \tilde{t} \tilde{t}}^{2} \tilde{G}_{\tilde{t} \tilde{t}}(\hat{s})} \\
& \times C_{H \tilde{t} \tilde{t}} \tilde{G}_{\tilde{t} \tilde{t}}(\hat{s}) C_{\gamma \gamma \tilde{t} \tilde{t} .}
\end{aligned}
$$

There is a contribution in which the initial $g g$ pair transitions to a stop-antistop pair, which transitions to a Higgs boson, which transitions to a $\gamma \gamma$ pair:

$$
\begin{aligned}
A_{3}(g g \rightarrow \gamma \gamma)= & C_{g \tilde{g} \tilde{t} \tilde{t}} \tilde{G}_{\tilde{t} \tilde{t}}(\hat{s}) C_{H \tilde{t} \tilde{t}} \\
& \times \frac{i}{\hat{s}-m_{H}^{2}+i m_{H} \Gamma_{H}-i C_{H \tilde{t} \tilde{t}}^{2} \tilde{G}_{\tilde{t} \tilde{t}}(\hat{s})} C_{\gamma \gamma H} .
\end{aligned}
$$

There is a contribution in which the initial $g g$ pair transitions to a stop-antistop pair, which transitions to a Higgs boson, which transitions to a stop-antistop pair, which transitions to a $\gamma \gamma$ pair:

$$
A_{4}(g g \rightarrow \gamma \gamma)=C_{g \tilde{g} \tilde{t} \tilde{G}} \tilde{G}_{\tilde{t} \tilde{t}}(\hat{s}) C_{H \tilde{t} \tilde{t}} \frac{i}{\hat{s}-m_{H}^{2}+i m_{H} \Gamma_{H}-i C_{H \tilde{t} \tilde{t}}^{2} \tilde{G}_{\tilde{t} \tilde{t}}(\hat{s})} C_{H \tilde{t} \tilde{t}} \tilde{G}_{\tilde{t} \tilde{t}}(\hat{s}) C_{\gamma \tilde{t} \tilde{t}}
$$

Finally, there is a contribution that does not involve the Higgs boson:

$$
A_{5}(g g \rightarrow \gamma \gamma)=C_{g g \tilde{t} \tilde{t}} \tilde{G}_{\tilde{t} \tilde{t}}(\hat{s}) C_{\gamma \gamma \tilde{t} \tilde{t}} .
$$

We note that the amplitudes $A_{4}$ and $A_{5}$ can be combined to give a simpler expression:

$$
\begin{aligned}
A_{4}^{\prime}(g g \rightarrow \gamma \gamma)= & A_{4}(g g \rightarrow \gamma \gamma)+A_{5}(g g \rightarrow \gamma \gamma) \\
= & C_{g g \tilde{t} \tilde{t}} \frac{1}{\tilde{G}_{\tilde{t} \tilde{t}}^{-1}(\hat{s})-C_{H \tilde{t} \tilde{t}}^{2} S_{H}(\hat{s})} C_{\gamma \gamma \tilde{t} \tilde{t}} \\
= & C_{g \tilde{t} \tilde{t} \tilde{G}} \tilde{G}_{\tilde{t} \tilde{t}}(\hat{s}) \\
& \times \frac{\hat{s}-m_{H}^{2}+i m_{H} \Gamma_{H}}{\hat{s}-m_{H}^{2}+i m_{H} \Gamma_{H}-i C_{H \tilde{t} \tilde{t}}^{2} \tilde{G}_{\tilde{t} \tilde{t}}(\hat{s})} C_{\gamma \gamma \tilde{t} \tilde{t}}
\end{aligned}
$$

The form of $A_{4}^{\prime}(g g \rightarrow \gamma \gamma)$ in Eq. (29b) is the same as the form of $A_{1}(g g \rightarrow \gamma \gamma)$ in Eq. (23b), but with the roles of the Higgs boson and the stop-antistop pair interchanged.
We also note that the total amplitude

$$
A_{\mathrm{tot}}(g g \rightarrow \gamma \gamma)=\sum_{i=1}^{5} A_{i}(g g \rightarrow \gamma \gamma)
$$

can be obtained from the matrix expression

$$
\begin{aligned}
& A_{\mathrm{tot}}(g g \rightarrow \gamma \gamma) \\
& =\left(\begin{array}{ll}
C_{g g H} & C_{g g \tilde{t} \tilde{t}}
\end{array}\right)\left(\begin{array}{cc}
S_{H}^{-1}(\hat{s}) & -C_{H \tilde{t} \tilde{t}} \\
-C_{H \tilde{t} \tilde{t}} & \tilde{G}_{\tilde{t} \tilde{t}}^{-1}(\hat{s})
\end{array}\right)^{-1}\left(\begin{array}{c}
C_{\gamma \gamma H} \\
C_{\gamma \gamma \tilde{t} \tilde{t}}
\end{array}\right) \\
& =\left(\begin{array}{ll}
C_{g g H} & C_{g \tilde{t} \tilde{t}}
\end{array}\right)\left(\begin{array}{cc}
\tilde{G}_{\tilde{t} \tilde{t}}^{-1}(\hat{s}) & C_{H \tilde{t} \tilde{t}} \\
C_{H \tilde{t} \tilde{t}} & S_{H}^{-1}(\hat{s})
\end{array}\right)\left(\begin{array}{c}
C_{\gamma \gamma H} \\
C_{\gamma \gamma \tilde{t} \tilde{t}}
\end{array}\right) \\
& \times \frac{1}{S_{H}^{-1}(\hat{s}) \tilde{G}_{\tilde{t} \tilde{t}}^{-1}(\hat{s})-C_{H \tilde{t} \tilde{t}}^{2}},
\end{aligned}
$$

where the matrix whose inverse is taken in Eq. (31a) is $(-i)$ times the effective Hamiltonian for the Higgs-stop-antistop system. The expression in Eq. (31a) is a generalization of Eq. (7) in Ref. [15]. 


\section{Coulomb-Schrödinger Green's function}

The stop-antistop Green's function $G_{\tilde{t} \tilde{t}}(\hat{s})$ can be computed at the leading nontrivial order in $v$, by allowing the stop and antistop to interact only through the potential of the leading order in $v$, which is called the static potential. One could take the static potential from lattice data, which are well described by a Coulomb-plus-linear potential (Cornell potential [40]) with a roll-off to a flat potential above the stop-antistop threshold. The Green's function corresponding to such a potential could, in principle, be evaluated numerically. In this paper, we choose instead to deal with a completely analytic Green's function, which, we believe, illustrates the qualitative features of the Higgsstop-antistop system. In particular, we make use of a modified Coulomb-Schrödinger Green's function, which we describe in detail below.

We obtain the relationship between $G_{\tilde{t} \tilde{t}}(\hat{s})$ and the Schrödinger Green's function as follows. Potential interactions are independent of the relative momentum $p_{0}$ of the stop quark and the antistop quark. Therefore, we integrate the effective-field-theory stop and antistop propagators over $p_{0}$ to obtain

$$
\begin{aligned}
\int \frac{d p_{0}}{2 \pi} \frac{i}{2 m_{\tilde{t}}\left(p_{0}+E / 2\right)-\mathbf{p}^{2}+i m_{\tilde{t}} \Gamma_{\tilde{t}}+i \epsilon} \frac{i}{-2 m_{\tilde{t}}\left(p_{0}+E / 2\right)-\mathbf{p}^{2}+i m_{\tilde{t}} \Gamma_{\tilde{t}}+i \epsilon} \\
\quad=\frac{i}{4 m_{\tilde{t}}^{2}} \frac{1}{E-\frac{p^{2}}{2 m_{\tilde{t}}}+i \Gamma_{\tilde{t}}+i \epsilon}=\frac{-i}{4 m_{\tilde{t}}^{2}} G_{\mathrm{S}}^{(0)}\left(E+i \Gamma_{\tilde{t}}, \boldsymbol{p}\right),
\end{aligned}
$$

where $\Gamma_{\tilde{t}}$ is the stop-quark width and $G_{\mathrm{S}}^{(0)}(E, \boldsymbol{p})$ is the Schrödinger propagator (Schrödinger Green's function in the absence of interactions). Hence, we conclude that, in the case of a Coulomb potential,

$$
G_{\tilde{t} \tilde{t}}(\hat{s})=\frac{-i}{4 m_{\tilde{t}}^{2}} G_{\mathrm{C}-\mathrm{S}}\left(0,0, E+i \Gamma_{\tilde{t}}\right)
$$

where $G_{\mathrm{C}-\mathrm{S}}\left(0,0, E+i \Gamma_{\tilde{t}}\right)$ is the Coulomb-Schrödinger Green's function evaluated at zero spatial separation between the initial stop and antistop and zero spatial separation between the final stop and antistop.

In the $\overline{\mathrm{MS}}$ scheme, $G_{\mathrm{C}-\mathrm{S}}\left(0,0, E+i \Gamma_{\tilde{t}}\right)$ is given by $[41,42]$

$$
\begin{aligned}
G_{\mathrm{C}-\mathrm{S}}(0,0, \tilde{E})= & \frac{\alpha_{s} C_{F}}{4 \pi} m_{\tilde{t}}^{2}\left[-\frac{1}{2 \lambda}-\frac{1}{2} \log \left(\frac{-4 m_{\tilde{t}} \tilde{E}}{\mu^{2}}\right)\right. \\
& \left.+\frac{1}{2}+\sum_{n=1}^{\infty} \frac{1}{n(n / \lambda-1)}\right] \\
= & \frac{\alpha_{s} C_{F}}{4 \pi} m_{\tilde{t}}^{2}\left[-\frac{1}{2 \lambda}-\frac{1}{2} \log \left(\frac{-4 m_{\tilde{t}} \tilde{E}}{\mu^{2}}\right)\right. \\
& \left.+\frac{1}{2}-\gamma_{\mathrm{E}}-\psi(1-\lambda)\right],
\end{aligned}
$$

where

$$
\begin{gathered}
\tilde{E}=E+i \Gamma_{\tilde{t}}, \\
\lambda \equiv \frac{\alpha_{s} C_{F}}{\sqrt{-4 \tilde{E} / m_{\tilde{t}}}},
\end{gathered}
$$

$\mu$ is the $\overline{\mathrm{MS}}$ scale, $\gamma_{\mathrm{E}}$ is Euler's constant, and $\psi(1-\lambda)$ is the digamma function. We take $\mu=m_{\tilde{t}}$.

The first and second terms in Eq. (34a) correspond to zero and one Coulomb interaction, respectively. These are the only contributions that are ultraviolet divergent and that, therefore, depend on the renormalization scheme. The sum in Eq. (34a) contains the contributions involving two or more Coulomb interactions. When $\Gamma_{\tilde{t}}=0$, the $n$th term in the sum contains the pole $\left|\psi_{n}(0)\right|^{2} /\left(E_{n}-E\right)$, where $E_{n}$ is the energy of the $n$th bound state and $\psi_{n}(0)$ is the wave function at the origin of the $n$th bound state. The $n$th term in the sum also contains nonpole contributions. It is best not to separate the pole and nonpole contributions, as either of them alone produces a spurious logarithmic singularity at $E=0$ (threshold).

In this work, we wish to capture the essential features of the QCD Green's function, which we expect to contain only a few bound-state poles below threshold. Therefore, we modify $G_{\mathrm{C}-\mathrm{S}}(0,0, E)$ by retaining only a few terms in the sum in Eq. (34a). We expect that, for large $m_{\tilde{t}}$, the lowest-lying bound states will be given approximately by the bound states of the Coulomb potential, and so the modified Coulomb Green's function should give a qualitative description of the system. ${ }^{7}$

\footnotetext{
${ }^{6}$ Another reasonable choice is $\mu=2 m_{\tilde{t}}$, which would shift the expressions in square brackets in Eqs. (34a) and (34b) by $-\log 2$. We believe that such a shift, which is small in comparison with the term $1 / 2$ in the expressions in square brackets, would have no qualitative effect on our results.

${ }^{7}$ Lattice measurements of the static quark-antiquark potential [43], which is identical to the static squark-antisquark potential, suggest that the static potential is predominantly Coulombic at short distances, as would be expected from asymptotic freedom. A recent lattice calculation [44] indicates that the stoponium groundstate wave functions at the origin, for $100 \mathrm{GeV} \leq m_{\tilde{t}} \leq 750 \mathrm{GeV}$, may have values that are substantially larger than those that are obtained from potentials that match QCD perturbation theory at short distances [45]. There is not, as yet, an independent confirmation of this surprising result.
} 
In computing the Coulomb Green's function, we set $\alpha_{s}$ to a constant by requiring that

$$
|\mathbf{p}|=v m_{\tilde{t}}=\alpha_{s}\left(v m_{\tilde{t}}\right) m_{\tilde{t}} .
$$

This equation should be approximately valid for the lowlying bound states. It yields $\alpha_{s} \approx 0.13$.

\section{E. Higgs-boson form factors}

The perturbative form factor for the coupling of the Higgs boson to diphotons through a stop loop is given by

$$
F(H \rightarrow \tilde{t} \tilde{t} \rightarrow \gamma \gamma)=C_{\gamma \gamma H}+C_{H \tilde{t} \tilde{t}} \tilde{G}_{\tilde{t} \tilde{t}} C_{\gamma \gamma \tilde{t} \tilde{t}} .
$$

The amplitude $A_{1}+A_{2}$ is proportional to this form factor, and, hence, this form factor is built into our formalism. There is a similar form factor for the Higgs coupling to two gluons through a stop loop:

$$
F(H \rightarrow \tilde{t} \tilde{t} \rightarrow g g)=C_{g g H}+C_{H \tilde{t} \tilde{t}} \tilde{G}_{\tilde{t} \tilde{t}} C_{g g \tilde{t} \tilde{t}} .
$$

The amplitude $A_{1}+A_{3}$ is proportional to this form factor, and, so, this form factor is also built into our formalism. The amplitude $A_{4}$ is proportional to the cross term between the Higgs-to-diphoton form factor and the Higgs-todigluon form factor:

$$
F(H \rightarrow \tilde{t} \tilde{t} \rightarrow \gamma \gamma g g)=C_{g g \tilde{t} \tilde{t}} C_{H \tilde{t} \tilde{t}}^{2} \tilde{G}_{\tilde{t} \tilde{t}}^{2} C_{\gamma \gamma \tilde{t} \tilde{t}} .
$$

The factors $\tilde{G}_{\tilde{t} \tilde{t}}$ in the second terms of the form factors in Eqs. (36) and (37) and in the form-factor contribution in Eq. (38) give enhancements of the total amplitude when $\hat{s}$ is near a threshold peak in $\tilde{G}_{\tilde{t} \tilde{t}}$. Such enhancements are already present in the perturbative calculations that make use of the first and second terms of the Coulomb Green's function [Eq. (34a)], and they become stronger when one takes into account the additional effects that are associated with the stop-antistop bound states [16]. However, as we will illustrate in detail in Sec. III, when the Higgs mass is close to stop-antistop threshold, the effect of Higgs-stopantistop mixing is to displace the physical mass peaks away from threshold. Consequently, when the full effects of Higgs-stop-antistop mixing are taken into account, this enhancement effect is not operative.

\section{CASE OF A SINGLE BREIT-WIGNER RESONANCE IN THE STOP-ANTISTOP GREEN'S FUNCTION}

We now discuss the situation in which we model $\tilde{G}_{\tilde{t} \tilde{t}}$ with the simplified form of a Breit-Wigner resonance. ${ }^{8}$ This

\footnotetext{
${ }^{8}$ This simplified model has also been considered in Ref. [6]. In Ref. [46], this model was used to investigate the effects of mixing of a light pseudoscalar particle with $\eta_{b}(n)$ states in the context of decays of the pseudoscalar particle to the $\eta_{b}(n)$ states.
}

is the only modification to the effective theory that we make. In particular, we use the formulas for the shortdistance coefficients that are given above.

\section{A. Structure of the Breit-Wigner amplitude}

We consider the situation in which $\tilde{G}_{\tilde{t} \tilde{t}}$ is given by

$$
\tilde{G}_{\tilde{t} \tilde{t}}=N_{\tilde{t} \tilde{t}}^{2} S_{\tilde{t} \tilde{t}}(\hat{s})
$$

where

$$
S_{\tilde{t} \tilde{t}}(\hat{s})=\frac{i}{\hat{s}-m_{\tilde{t} \tilde{t}}^{2}+i m_{\tilde{t} \tilde{t}} \Gamma_{\tilde{t} \tilde{t}}}
$$

$m_{\tilde{t} \tilde{t}}$ is the bound-state mass, $\Gamma_{\tilde{t} \tilde{t}}$ is the bound-state width,

$$
N_{\tilde{t} \tilde{t}}^{2}=|\psi(0)|^{2} / m_{\tilde{t}},
$$

and $\psi(0)$ is the bound-state wave function at the origin. The bound-state width is given by

$$
\Gamma_{\tilde{t} \tilde{t}}=2 \Gamma_{\tilde{t}}+\Gamma_{\tilde{t} \tilde{t} g g} .
$$

Here, $\Gamma_{\tilde{t} \tilde{t} g g}$, is the width of the bound state to two gluons:

$$
\Gamma_{\tilde{t} \tilde{t} g g}=\frac{1}{\left(2 m_{\tilde{t}}\right)^{2}} 2 \operatorname{Im} T_{\tilde{t} \tilde{t} \rightarrow g g \rightarrow \tilde{t} \tilde{t}}|\psi(0)|^{2}=\frac{4 \pi \alpha_{s}^{2}\left(m_{\tilde{t}}\right)|\psi(0)|^{2}}{3 m_{\tilde{t}}^{2}} .
$$

For the Coulomb ground state, $|\psi(0)|^{2}=8 \alpha_{s}^{3}\left(m_{\tilde{t}} v\right) m_{\tilde{t}}^{3} /(27 \pi)$.

Now, we can write Eq. (31a) as

$$
\begin{aligned}
A_{\mathrm{tot}}(g g \rightarrow \gamma \gamma)= & \left(\begin{array}{ll}
C_{g g H} & \hat{C}_{g \tilde{g} \tilde{t} \tilde{t}}
\end{array}\right)\left(\begin{array}{cc}
S_{H}^{-1}(\hat{s}) & -\hat{C}_{H \tilde{t} \tilde{t}} \\
-\hat{C}_{H \tilde{t} \tilde{t}} & S_{\tilde{t} \tilde{t}}^{-1}(\hat{s})
\end{array}\right)^{-1} \\
& \times\left(\begin{array}{c}
C_{\gamma \gamma H} \\
\hat{C}_{\gamma \gamma \tilde{t} \tilde{t}}
\end{array}\right),
\end{aligned}
$$

where $\quad \hat{C}_{g g \tilde{t} \tilde{t}}=N_{\tilde{t} \tilde{t}} C_{g g \tilde{t} \tilde{t}}, \quad \hat{C}_{\gamma \gamma \tilde{t} \tilde{t}}=N_{\tilde{t} \tilde{t}} C_{\gamma \gamma \tilde{t} \tilde{t}}$, and $\hat{C}_{H \tilde{t} \tilde{t}}=$ $N_{\tilde{t} \tilde{t}} C_{H \tilde{t} \tilde{t}}$. We can diagonalize the matrix in Eq. (42) by making use of a similarity transformation:

$$
\begin{gathered}
-i\left(\begin{array}{cc}
\hat{s}^{2}-m_{+}^{2}+i m_{+} \Gamma_{+} & 0 \\
0 & \hat{s}^{2}-m_{-}^{2}+i m_{-} \Gamma_{-}
\end{array}\right) \\
=S(\theta)^{-1}\left(\begin{array}{cc}
S_{H}^{-1}(\hat{s}) & \hat{C}_{H \tilde{t} \tilde{t}} \\
\hat{C}_{H \tilde{t} \tilde{t}} & S_{\tilde{t} \tilde{t}}^{-1}(\hat{s})
\end{array}\right) S(\theta),
\end{gathered}
$$

which implies that 


$$
\begin{aligned}
& A_{\mathrm{tot}}(g g \rightarrow \gamma \gamma) \\
& \quad=\left(\begin{array}{ll}
C_{g g+} & C_{g g-}
\end{array}\right)\left(\begin{array}{cc}
\frac{i}{\hat{s}^{2}-m_{+}^{2}+i m_{+} \Gamma_{+}} & 0 \\
0 & \frac{i}{\hat{s}^{2}-m_{-}^{2}+i m_{-} \Gamma_{-}}
\end{array}\right)\left(\begin{array}{c}
C_{\gamma \gamma+} \\
C_{\gamma \gamma-}
\end{array}\right),
\end{aligned}
$$

where

$$
\left(\begin{array}{c}
C_{\gamma \gamma+} \\
C_{\gamma \gamma-}
\end{array}\right)=S^{-1}(\theta)\left(\begin{array}{c}
C_{\gamma \gamma H} \\
\hat{C}_{\gamma \gamma \tilde{t} \tilde{t}}
\end{array}\right)
$$

and

$$
\left(\begin{array}{ll}
C_{g g+} & C_{g g-}
\end{array}\right)=\left(\begin{array}{ll}
C_{g g H} & \hat{C}_{g g \tilde{t} \tilde{t}}
\end{array}\right) S(\theta) .
$$

The masses and widths are given by

$$
\begin{aligned}
& m_{ \pm}^{2}-i m_{ \pm} \Gamma_{ \pm} \equiv \frac{1}{2}\left(m_{H}^{2}-i m_{H} \Gamma_{H}+m_{\tilde{t} \tilde{t}}^{2}-i m_{\tilde{t} \tilde{t}} \Gamma_{\tilde{t} \tilde{t}}\right) \\
& \pm \frac{1}{2} \sqrt{\left(m_{H}^{2}-i m_{H} \Gamma_{H}-m_{\tilde{t} \tilde{t}}^{2}+i m_{\tilde{t} \tilde{t}} \Gamma_{\tilde{t} \tilde{t} \tilde{t}}\right)^{2}+4\left|\hat{C}_{H \tilde{t} \tilde{t}}\right|^{2}} .
\end{aligned}
$$

(Recall that, in our definition, $\hat{C}_{H \tilde{t} \tilde{t}}$ is purely imaginary.) Note that these values of $m_{ \pm}-i m_{ \pm} \Gamma_{ \pm}$correspond precisely to the values of $\hat{s}$ at which the denominators in $A_{1} \ldots A_{4}$ [Eqs. (23)-(27) and Eq. (31b)] vanish.

Approximate expressions for the masses and widths are

$$
m_{ \pm}^{2} \approx \frac{1}{2}\left(m_{H}^{2}+m_{\tilde{t} \tilde{t}}^{2}\right) \pm \frac{1}{2} \Delta,
$$

$$
m_{ \pm} \Gamma_{ \pm} \approx \frac{1}{2}\left[m_{H} \Gamma_{H}+m_{\tilde{t} \tilde{t}} \Gamma_{\tilde{t} \tilde{t}} \pm\left(m_{H} \Gamma_{H}-m_{\tilde{t} \tilde{t}} \Gamma_{\tilde{t} \tilde{t}}\right) \frac{m_{H}^{2}-m_{\tilde{t} \tilde{t}}^{2}}{\Delta}\right],
$$

where

$$
\Delta \equiv \sqrt{\left(m_{H}^{2}-m_{\tilde{t} \tilde{t}}^{2}\right)^{2}+4\left|\hat{C}_{H \tilde{t} \tilde{t}}\right|^{2}} .
$$

In the approximate forms in Eq. (46), we have neglected terms of higher order in $\left(m_{H} \Gamma_{H}-m_{\tilde{t} \tilde{t}} \Gamma_{\tilde{t} \tilde{t}}\right)^{2} / \Delta^{2}$, which is less than $2 \%$ for the values of $m_{H} \Gamma_{H}, m_{\tilde{t} \tilde{t}} \Gamma_{\tilde{t} \tilde{t}}$, and $\hat{C}_{H \tilde{t} \tilde{t}}^{2}$ that we use in our cross-section calculations in Sec. III B.

The matrix $S(\theta)$ is given by

$$
S(\theta)=\left(\begin{array}{cc}
\cos \theta & -\sin \theta \\
\sin \theta & \cos \theta
\end{array}\right),
$$

where

$$
\tan \theta=\frac{2\left|\hat{C}_{H \tilde{t} \tilde{t}}\right|}{\sqrt{\left(m_{H}^{2}-i m_{H} \Gamma_{H}-m_{\tilde{t} \tilde{t}}^{2}+i m_{\tilde{t} \tilde{t}} \Gamma_{\tilde{t} \tilde{t}}\right)^{2}+4\left|\hat{C}_{H \tilde{t} \tilde{t}}\right|^{2}}-\left(m_{H}^{2}-i m_{H} \Gamma_{H}-m_{\tilde{t} \tilde{t}}^{2}+i m_{\tilde{t} \tilde{t}} \Gamma_{\tilde{t} \tilde{t} \tilde{t}}\right)} \approx \frac{2\left|\hat{C}_{H \tilde{t} \tilde{t}}\right|}{\Delta-\left(m_{H}^{2}-m_{\tilde{t} \tilde{t}}^{2}\right)+i \delta},
$$

with

$$
\delta=\left(m_{H} \Gamma_{H}-m_{\tilde{t} \tilde{t}} \Gamma_{\tilde{t} \tilde{t}}\right)\left(1-\frac{m_{H}^{2}-m_{\tilde{t} \tilde{t}}^{2}}{\Delta}\right) .
$$

In the approximation in Eq. (48a), we have again neglected terms of higher order in $\left(m_{H} \Gamma_{H}-m_{\tilde{t} \tilde{t}} \Gamma_{\tilde{t} \tilde{t}}\right)^{2} / \Delta^{2}$.

Note that $m_{+}^{2}$ and $m_{-}^{2}$ are always centered at the average of $m_{H}^{2}$ and $m_{\tilde{t} \tilde{t}}^{2}$ and separated from each other by a nonzero amount, namely, $\Delta$. When $2\left|\hat{C}_{H \tilde{t} \tilde{t}}\right|$ is small in comparison with $\left|m_{H}^{2}-m_{\tilde{t} \tilde{t}}^{2}\right|, \theta$ approaches 0 or $\pi / 2$, and the masses and widths approach their original values. On the other hand, when $\left|m_{H}^{2}-m_{\tilde{t} \tilde{t}}^{2}\right|$ is negligible in comparison with $2\left|\hat{C}_{H \tilde{t} \tilde{t}}\right|$, mixing is maximal, and $\theta$ is very close to $\pi / 4 .^{9}$ In this case of maximal mixing, $\Delta$ approaches its minimum value, $2\left|\hat{C}_{H \tilde{t} \tilde{t}}\right|$, and

$$
m_{ \pm}^{2} \approx \frac{1}{2}\left(m_{H}^{2}+m_{\tilde{t} \tilde{t}}^{2}\right) \pm\left|\hat{C}_{H \tilde{t} \tilde{t}}\right|
$$

\footnotetext{
${ }^{9}$ For the values of the theory parameters that are given in Sec. III B, the real part of $\theta$ is within $1 \%$ of $\pi / 4$, while the imaginary part of $\theta$ is less than $7 \%$.
}

In the case of maximal mixing, the widths are given by

$$
m_{ \pm} \Gamma_{ \pm} \approx \frac{1}{2}\left(m_{H} \Gamma_{H}+m_{\tilde{t} \tilde{t}} \Gamma_{\tilde{t} \tilde{t}}\right) .
$$

That is, the widths $\Gamma_{+}$and $\Gamma_{-}$become approximately the average of $\Gamma_{H}$ and $\Gamma_{\tilde{t} \tilde{t}}$.

It is illuminating to compute the physical mass separations and widths for the values of the theory parameters that we use in our cross-section calculations in Sec. III B. Even in the case of maximal mixing, for which the separation between $m_{+}$and $m_{-}$is minimal, that separation is still substantial: about $4.7 \mathrm{GeV}$ for $\kappa=1$ and about $37.4 \mathrm{GeV}$ for $\kappa=8$. These separations are much greater than the widths of the Breit-Wigner resonances, which are $\Gamma_{H} \approx 1.2 \mathrm{GeV}, \Gamma_{\tilde{t} \tilde{t}} \approx 0.3 \mathrm{MeV}$ for $\Gamma_{\tilde{t}}=0.1 \mathrm{MeV}$, and $\Gamma_{\tilde{t} \tilde{t}} \approx 0.2 \mathrm{GeV}$ for $\Gamma_{\tilde{t}}=0.1 \mathrm{GeV}$. Consequently, the mass separations are also much greater than $\Gamma_{ \pm}$. That is, the physical resonances are well separated for any value of $m_{H}$ relative to $m_{\tilde{t} \tilde{t}}$.

In Sec. II E, we discussed the threshold enhancements that are present in the form factors for the Higgs couplings to $g g$ and $\gamma \gamma$. We now see that those enhancements are 
rendered inoperative because, when $m_{H}$ approaches the stop-antistop threshold, the physical masses are displaced from threshold by a sizable amount. In the next section, we will provide a detailed numerical analysis of this effect.

\section{B. Qualitative features of the Breit-Wigner cross section}

In this section, we present numerical results for the shortdistance coefficients and also for cross sections at the LHC at a CM energy $\sqrt{s}$ of $13 \mathrm{TeV}$. We note that the $g g$ contribution to the total cross section is given by the expression

$$
\sigma_{\mathrm{tot}}=\frac{1}{128 \pi s} \int_{\sqrt{\hat{s}_{\min }}}^{\sqrt{\hat{s}_{\max }}} \frac{d \sqrt{\hat{s}}}{\sqrt{\hat{s}}} \int_{\hat{s} / s}^{1} \frac{d x}{x} f_{g}\left(\frac{\hat{s}}{x s}\right) f_{g}(x)\left|A_{\mathrm{tot}}(\hat{s})\right|^{2},
$$

where $f_{g}$ is the gluon distribution and $\sqrt{\hat{s}_{\min }}$ and $\sqrt{\hat{s}_{\max }}$ are the lower and upper limits, respectively, of the range in $\sqrt{\hat{s}}$ that includes all relevant contributions to the cross section. In the computations of $\sigma_{\text {tot }}$ in the remainder of this paper, we take $\sqrt{\hat{s}_{\min }}=600 \mathrm{GeV}$ and $\sqrt{\hat{s}_{\max }}=900 \mathrm{GeV}$.

As we have already mentioned, the Breit-Wigner resonances are always well separated in comparison to their widths. Therefore, we can approximate the cross section in Eq. (51) as a sum of the individual contributions of the two resonances. If we also neglect the dependences of the gluon distributions on $\hat{s}$ over the width of the resonance, then we obtain the narrow-resonance approximation

$$
\begin{aligned}
\sigma_{\mathrm{tot}}= & \frac{1}{128 \pi s} \sum_{j= \pm} \int_{0}^{\infty} \frac{d \sqrt{\hat{s}}}{m_{j}}\left|\frac{i}{\hat{s}-m_{j}^{2}+i m_{j} \Gamma_{j}}\right|^{2} \\
& \times\left|C_{g g j} C_{\gamma \gamma j}\right|^{2} F\left(m_{j}\right)+O\left(\Gamma_{j} / m_{j}\right) \\
= & \frac{1}{256 s} \sum_{j= \pm} \frac{\left|C_{g g j} C_{\gamma \gamma j}\right|^{2}}{m_{j}^{3} \Gamma_{j}} F\left(m_{j}\right)+O\left(\Gamma_{j} / m_{j}\right),
\end{aligned}
$$

where $F\left(m_{j}\right)$ is the gluon flux factor:

$$
F\left(m_{j}\right)=\int_{m_{j}^{2} / s}^{1} \frac{d x}{x} f_{g}\left(\frac{m_{j}^{2}}{x s}\right) f_{g}(x) .
$$

As can be seen from Fig. $1, F\left(m_{j}\right)$ is a slowly varying function of $m_{j}$ over the range of interest. ${ }^{10}$ It has only a small effect on the shape of the cross section as a function of $m_{H}$, which is determined mainly by the dependences of the short-distance coefficients $C_{g g j}$ and $C_{\gamma \gamma j}$ and the resonance widths $\Gamma_{j}$ on $m_{H}$.

Note that the cross section in the narrow-resonance approximation varies inversely as the width $\Gamma_{j}$. In general,

\footnotetext{
${ }^{10}$ In the calculations of cross sections in this paper, we make use of the CTEQ6M parton distribution functions [47].
}

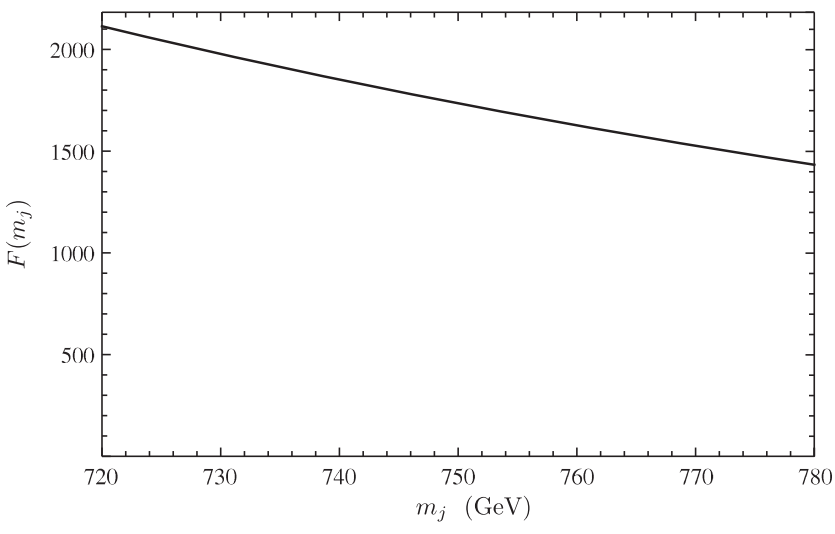

FIG. 1. The gluon flux factor $F\left(m_{j}\right)$.

the cross-section contribution of a Breit-Wigner resonance is proportional to the square of the absolute value of the maximum height of the amplitude times the width of the resonance. In the Breit-Wigner model of this section, the inverse of the diagonal matrix in Eq. (43) has elements whose maximum absolute values are equal to $1 /\left(m_{j} \Gamma_{j}\right)$. Therefore, the effect of this matrix factor on the cross section can be characterized completely in terms of the widths of the physical resonances. We use this characterization for the remainder of this section. As we will explain in Sec. III C, in case of the Coulomb-Schrödinger model, the corresponding diagonal matrix is more complicated, and its effect on the cross section cannot be characterized completely in terms of the widths of the physical resonances.

We discuss below the behavior of the cross section for four cases: $\kappa=1, \Gamma_{\tilde{t}}=0.1 \mathrm{MeV} ; \kappa=1, \Gamma_{\tilde{t}}=0.1 \mathrm{GeV}$; $\kappa=8, \Gamma_{\tilde{t}}=0.1 \mathrm{MeV}$; and $\kappa=8, \Gamma_{\tilde{t}}=0.1 \mathrm{GeV}$. The $\kappa$ value of 8 is near the upper limit of the values of $\kappa$ that are allowed by unitarity constraints [48]. As we have mentioned, in order to make contact with previous numerical work, we take the stop mass to be $m_{\tilde{t}}=375 \mathrm{GeV}$. We also use the input values $m_{b}\left(2 m_{\tilde{t}}\right)=2.46 \mathrm{GeV}$, $m_{t}\left(2 m_{\tilde{t}}\right)=149.95 \mathrm{GeV}$, where $m_{t}\left(2 m_{\tilde{t}}\right)$ and $m_{b}\left(2 m_{\tilde{t}}\right)$ are the $\overline{\mathrm{MS}}$ running masses at the scale $2 m_{\tilde{t}}$. We estimate the Higgs width by computing its widths to top- and bottom-quark pairs through order $\alpha_{s}[49,50]^{11}$ and its width to a $\tau$-lepton pair at the Born level, using the Higgs couplings to top-quark, bottom-quark, and $\tau$-lepton pairs that occur in the decoupling limit of large Higgs mass [13]. We will make use of an intermediate value of $\tan \beta$, setting $\tan \beta=\sqrt{m_{t}\left(2 m_{\tilde{t}}\right) / m_{b}\left(2 m_{\tilde{t}}\right)}$. At this value of $\tan \beta$, the Higgs decay width into third generation fermions is minimized, and our estimate is $\Gamma_{H} \simeq 1.2 \mathrm{GeV}$.

\footnotetext{
${ }^{11}$ We have converted the result in Ref. [50], which is expressed in terms of quark pole mass, to an expression in terms of the quark modified-minimal-subtraction $(\overline{\mathrm{MS}})$ mass by adding $2+$ $(3 / 2) \log \left[4 /\left(1-\beta^{2}\right)\right]$ to $\Delta_{H}$ in Eq. (2.26) of Ref. [50].
} 

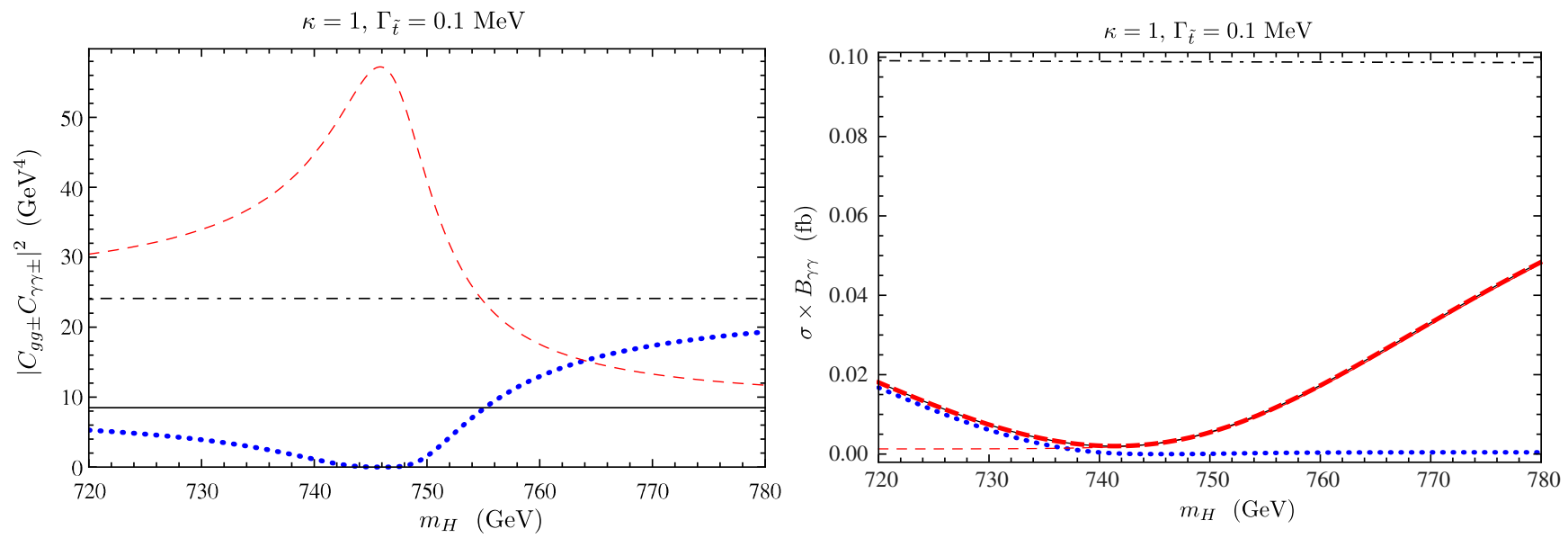

FIG. 2. Numerical results for the case in which the stop-antistop Green's function is given by a Breit-Wigner resonance for $\kappa=1$ and $\Gamma_{\tilde{t}}=0.1 \mathrm{MeV}$. Quantities are presented as functions of $m_{H}$. The left panel shows the products of absolute squares of short-distance coefficients: $\left|C_{g g+} C_{\gamma \gamma+}\right|^{2}$ (dotted, blue line), $\left|C_{g g-} C_{\gamma \gamma-}\right|^{2}$ (narrow, dashed, orange line), $\left|C_{g g \tilde{t} \tilde{t}} C_{\gamma \gamma \tilde{t} \tilde{t}}\right|^{2}$ (solid, black line), and $\left|C_{g g H} C_{\gamma \gamma H}\right|^{2}$ (dash-dotted, black line). The right panel shows cross sections times branching ratios into $\gamma \gamma$ : contribution in the narrow-resonance approximation of the larger-mass eigenstate (dotted, blue line), contribution in the narrow-resonance approximation of the smaller-mass eigenstate (narrow, dashed, orange line), sum of the contributions in the narrow-resonance approximation (thick, dashed, red line), exact cross section times branching ratio into $\gamma \gamma$ (thin, black line), and exact cross section times branching ratio into $\gamma \gamma$ in the absence of mixing (dash-dotted, black line).

\section{1. $\kappa=1, \Gamma_{\tilde{t}}=0.1 \mathrm{MeV}$}

We consider first the case of weak Higgs-stop-antistop coupling, $\kappa=1$, and small stop width, $\Gamma_{\tilde{t}}=0.1 \mathrm{MeV}$.

Let us examine the effect of mixing on the short-distance coefficients. In the left panel of Fig. 2, we show $\left|C_{\gamma \gamma \pm} C_{g g \pm}\right|^{2}$ as functions of $m_{H}$. We also show $\left|C_{\gamma \gamma \tilde{t} \tilde{t}} C_{g g \tilde{t} \tilde{t}}\right|^{2}$ and $\left|C_{\gamma \gamma H} C_{g g H}\right|^{2}$, so that one can judge the importance of the mixing effects. At small values of $m_{H}$, the lower-mass eigenstate corresponds to the Higgs boson, and the higher-mass eigenstate corresponds to the stopantistop bound state. This correspondence is reversed for large values of $m_{H}$. That is, at both large and small values of $m_{H}$, the upper line corresponds to the Higgs coefficients, and the lower line corresponds to the stop-antistop coefficients. Maximal mixing occurs when $m_{H}$ is equal to the stop-antistop bound-state mass $m_{\tilde{t} \tilde{t}}$, which is about $747 \mathrm{GeV}$. For large values of $\left|m_{H}-m_{\tilde{t} \tilde{t}}\right|$, the mixing angle decreases approximately as $1 /\left|m_{H}-m_{\tilde{t} \tilde{t}}\right|$. However, we remind the reader that the nonrelativistic approximation that we use in our calculations is valid only when $\left|m_{H}-m_{\tilde{t} \tilde{t}}\right| \ll m_{\tilde{t} \tilde{t}}$. The structure that appears in the quantities $\left|C_{g g \pm} C_{\gamma \gamma \pm}\right|^{2}$ is a consequence of the fact that the Higgs coefficients contain real and imaginary parts that are comparable in magnitude. The mixing then produces a complicated pattern of interference. We emphasize that the peak that appears in the upper line is not produced by a resonance or by a threshold enhancement from the Higgs to $g g$ or $\gamma \gamma$ form factors. Rather, it is entirely a consequence of interference effects in the short-distance coefficients.

Now, let us consider the behavior of the cross section as a function of $m_{H}$. In the right panel of Fig. 2, we show the contributions of the larger-mass eigenstate and the smallermass eigenstate to the cross section times the branching ratio into $\gamma \gamma$ in the narrow-resonance approximation, the sum of those contributions, and the exact cross section times the branching ratio into $\gamma \gamma$. We also show the exact cross section in the absence of mixing so that one can judge the importance of the mixing effects.

We can understand the qualitative features of the cross section times the branching ratio into $\gamma \gamma$ from the formula for the narrow-resonance approximation to the cross section in Eq. (52). As can be seen from Fig. 2, the factors $1 / \Gamma_{ \pm}$in Eq. (52a) have a strong effect on the shape of the cross section. When $m_{H}$ is small (large), the mixing is minimal, and $\Gamma_{+}\left(\Gamma_{-}\right)$is equal to $\Gamma_{\tilde{t} \tilde{t}} \approx 3 \mathrm{MeV}$. When $m_{H}$ approaches $m_{\tilde{t} \tilde{t}}$, the mixing becomes maximal, and $\Gamma_{ \pm} \approx\left(\Gamma_{H}+\Gamma_{\tilde{t} \tilde{t}}\right) / 2 \approx 0.6 \mathrm{GeV}$. The dependence of the cross section on $1 / \Gamma_{ \pm}$completely overwhelms the dependence of the cross section on the short-distance coefficients, resulting in the shape for the total cross section that is shown in the thick, dashed, red line in the right panel. The cross section times the branching ratio is suppressed at values of $m_{H}$ that are close to $m_{\tilde{t} \tilde{t}}$, owing to the increase in the width of the narrowest resonance from the stoponium width to the average of the stoponium and Higgs widths. Even at $m_{H}=720 \mathrm{GeV}$ and $m_{H}=780 \mathrm{GeV}$, where the mixing angles are fairly small, the effect of mixing on the width of the narrowest resonance leads to a considerable suppression of the values of the cross section times the branching fraction relative to the values in the absence of mixing. We note that the narrow-resonance approximation gives a result for the cross section that is very close to the exact result. (The line for the exact result is almost 

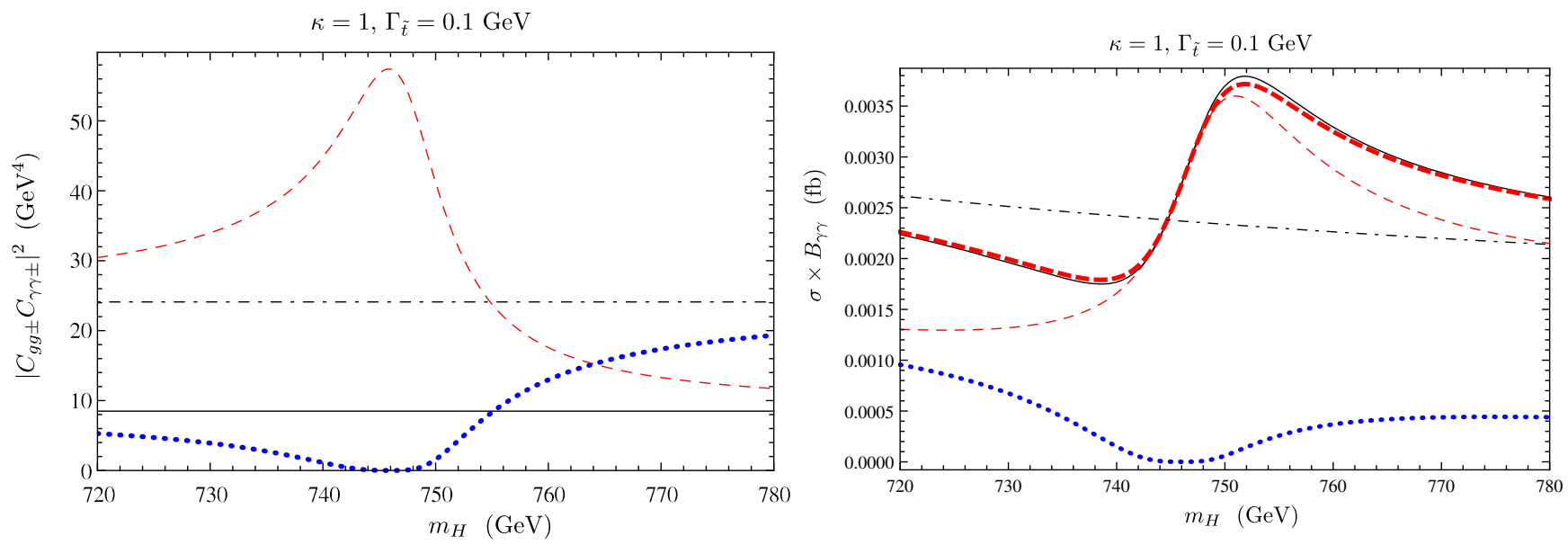

FIG. 3. Numerical results for the case in which the stop-antistop Green's function is given by a Breit-Wigner resonance for $\kappa=1$ and $\Gamma_{\tilde{t}}=0.1 \mathrm{GeV}$. Quantities are presented as functions of $m_{H}$. The left panel shows the products of absolute squares of short-distance coefficients: $\left|C_{g g+} C_{\gamma \gamma+}\right|^{2}$ (dotted, blue line), $\left|C_{g g-} C_{\gamma \gamma-}\right|^{2}$ (narrow, dashed, orange line), $\left|C_{g g \tilde{t} \tilde{t}} C_{\gamma \gamma \tilde{t} \tilde{t}}\right|^{2}$ (solid, black line), and $\left|C_{g g H} C_{\gamma \gamma H}\right|^{2}$ (dash-dotted, black line). The right panel shows cross sections times branching ratios into $\gamma \gamma$ : contribution in the narrow-resonance approximation of the larger-mass eigenstate (dotted, blue line), contribution in the narrow-resonance approximation of the smaller-mass eigenstate (narrow, dashed, orange line), sum of the contributions in the narrow-resonance approximation (thick, dashed, red line), exact cross section times branching ratio into $\gamma \gamma$ (thin, black line), and exact cross section times branching ratio into $\gamma \gamma$ in the absence of mixing (dash-dotted, black line).

completely obscured by the line for the narrow-resonanceapproximation result.)

\section{2. $\kappa=1, \Gamma_{\tilde{t}}=0.1 \mathrm{GeV}$}

Next, we consider the case of weak Higgs-stop-antistop coupling, $\kappa=1$, and large stop width, $\Gamma_{\tilde{t}}=0.1 \mathrm{GeV}$.

In Fig. 3, we display the values of $\left|C_{\gamma \gamma \pm} C_{g g \pm}\right|^{2}$ as functions of $m_{H}$. Again, we also show $\left|C_{\gamma \gamma \tilde{t} \tilde{t}} C_{g g \tilde{t} \tilde{t}}\right|^{2}$ and
$\left|C_{\gamma \gamma H} C_{g g H}\right|^{2}$, so that one can judge the importance of the mixing effects. As can be seen by comparing the left panel of Fig. 3 with the left panel of Fig. 2, the effect of mixing on the short-distance coefficients in the case of large stop width is essentially the same as in the case of small stop width. However, the stop-antistop width is now $\Gamma_{\tilde{t} \tilde{t}} \approx 0.2 \mathrm{GeV}$, which is not far from the Higgs width $\Gamma_{H} \approx 1.2 \mathrm{GeV}$. It follows that the width of the narrowest
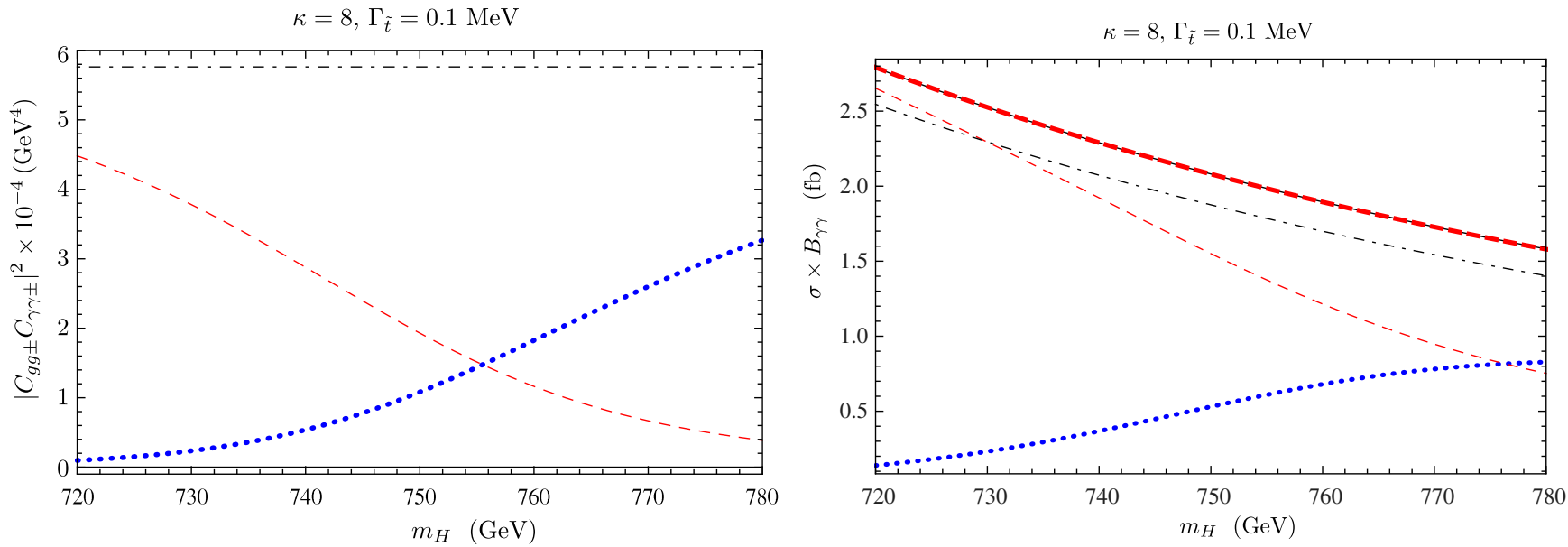

FIG. 4. Numerical results for the case in which the stop-antistop Green's function is given by a Breit-Wigner resonance for $\kappa=8$ and $\Gamma_{\tilde{t}}=0.1 \mathrm{MeV}$. Quantities are presented as functions of $m_{H}$. The left panel shows the products of absolute squares of short-distance coefficients: $\left|C_{g g+} C_{\gamma \gamma+}\right|^{2}$ (dotted, blue line), $\left|C_{g g-} C_{\gamma \gamma-}\right|^{2}$ (narrow, dashed, orange line), $\left|C_{g g \tilde{t} \tilde{t}} C_{\gamma \gamma \tilde{t} \tilde{t}}\right|^{2}$ (solid, black line), and $\left|C_{g g H} C_{\gamma \gamma H}\right|^{2}$ (dash-dotted, black line). The right panel shows cross sections times branching ratios into $\gamma \gamma$ : contribution in the narrow-resonance approximation of the larger-mass eigenstate (dotted, blue line), contribution in the narrow-resonance approximation of the smaller-mass eigenstate (narrow, dashed, orange line), sum of the contributions in the narrow-resonance approximation (thick, dashed, red line), exact cross section times branching ratio into $\gamma \gamma$ (thin, black line), and exact cross section times branching ratio into $\gamma \gamma$ in the absence of mixing (dash-dotted, black line). 
resonance goes from about $0.2 \mathrm{GeV}$ for minimal mixing to about $0.7 \mathrm{GeV}$ for maximal mixing, which is a much smaller range than in the case of a small stop width. Consequently, as can be seen from the right panel of Fig. 3, the effect of mixing on the resonance widths has a much less dramatic effect on the shape of the cross section times the branching ratio than in the small-stop-width case. The shape of the cross section times the branching ratio in the thick, dashed, red line now exhibits a peak that corresponds to the peak in the sum of the magnitudes of the products of short-distance coefficients. Comparing with the situation for small stop width, we see that the cross section times the branching ratio away from threshold is significantly smaller, owing to that fact that the stoponium width is now much larger. For the same reason, the cross section times the branching ratio away from threshold more closely approaches the cross section in the absence of mixing. Again, we note that the result from the narrow-resonance approximation for the cross section times the branching ratio into $\gamma \gamma$ agrees well with the exact result.

\section{3. $\kappa=8, \Gamma_{\tilde{t}}=0.1 \mathrm{MeV}$}

Next, we consider the case of strong Higgs-stop-antistop coupling, $\kappa=8$, and small stop width, $\Gamma_{\tilde{t}}=0.1 \mathrm{MeV}$.

As can be seen from the left panel of Fig. 4, the effect of mixing on the short-distance coefficients now produces only monotonic functions with no peaks or dips. This simple structure is attributable to the fact that $C_{H g g}$ and $C_{H \gamma \gamma}$ are dominated by their imaginary parts. Furthermore, as can be seen from the figure, the product of Higgs shortdistance coefficients is much larger in magnitude than the product of stop-antistop coefficients, and so the Higgs coefficients dominate at minimal mixing. At $m_{H}=$ $720 \mathrm{GeV}$ and $m_{H}=780 \mathrm{GeV}$, the effects on the shortdistance coefficients are still considerable, especially at $m_{H}=780 \mathrm{GeV}$. The stop-antistop width $\Gamma_{\tilde{t} \tilde{t}}$ at $m_{H}=$ $720 \mathrm{GeV}$ is about $0.24 \mathrm{GeV}$ and increases as $m_{H}$ approaches the stop-antistop threshold. Therefore, owing to the dominance of the Higgs short-distance coefficients, the stopantistop resonance does not contribute greatly to the cross section times the branching ratio. At maximal mixing, there are two resonances, whose widths are equal to about $\frac{1}{2} \Gamma_{H}$ and whose values of $\left|C_{g g \pm} C_{\gamma \gamma \pm}\right|^{2}$ are about $\frac{1}{2}\left|C_{g g H} C_{\gamma \gamma H}\right|^{2}$. Consequently, the cross section times the branching ratio changes very little from minimal mixing to maximal mixing, as can be seen in the right panel of Fig. 4. Even at $m_{H}=$ $720 \mathrm{GeV}$ and $m_{H}=780 \mathrm{GeV}$, the values of the cross section times the branching ratio lie somewhat above the values in the absence of mixing. The result from the narrowresonance approximation for the cross section is essentially featureless. We note that it agrees well with the exact result.

$$
\text { 4. } \kappa=8, \Gamma_{\tilde{t}}=0.1 \mathrm{GeV}
$$

Finally, we consider the case of strong Higgs-stop-antistop coupling, $\kappa=8$, and large stop width, $\Gamma_{\tilde{t}}=0.1 \mathrm{GeV}$.

As can be seen from the left panel of Fig. 5, the effect of mixing on the short-distance coefficients is essentially the same as in the case of $\kappa=8$ and $\Gamma_{\tilde{t}}=0.1 \mathrm{MeV}$ (Fig. 4). Again, owing to the dominance of the imaginary parts of $C_{H g g}$ and $C_{H \gamma \gamma}$, the effects of mixing on the short-distance coefficients result in a simple structure. Since the product of Higgs short-distance coefficients is much larger in
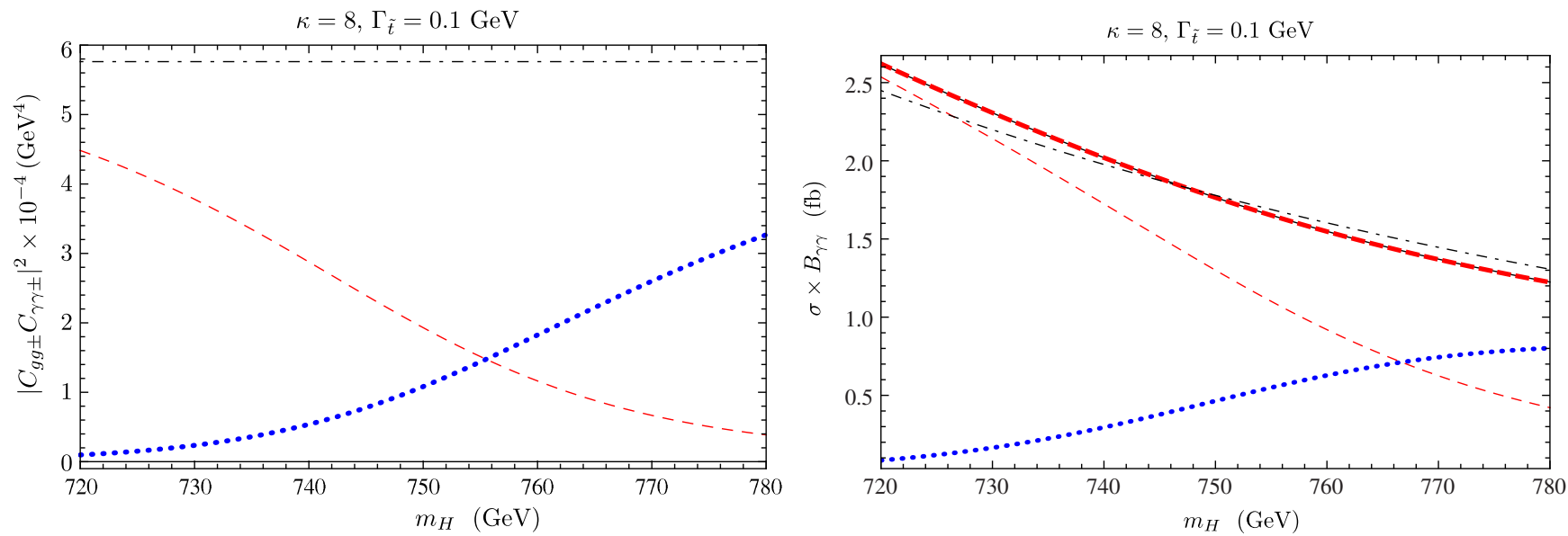

FIG. 5. Numerical results for the case in which the stop-antistop Green's function is given by a Breit-Wigner resonance for $\kappa=8$ and $\Gamma_{\tilde{t}}=0.1 \mathrm{GeV}$. Quantities are presented as functions of $m_{H}$. The left panel shows the products of absolute squares of short-distance coefficients: $\left|C_{g g+} C_{\gamma \gamma+}\right|^{2}$ (dotted, blue line), $\left|C_{g g-} C_{\gamma \gamma-}\right|^{2}$ (narrow, dashed, orange line), $\left|C_{g g \tilde{t} \tilde{t}} C_{\gamma \gamma \tilde{t} \tilde{t}}\right|^{2}$ (solid, black line), and $\left|C_{g g H} C_{\gamma \gamma H}\right|^{2}$ (dash-dotted, black line). The right panel shows cross sections times branching ratios into $\gamma \gamma$ : contribution in the narrow-resonance approximation of the larger-mass eigenstate (dotted, blue line), contribution in the narrow-resonance approximation of the smaller-mass eigenstate (narrow, dashed, orange line), sum of the contributions in the narrow-resonance approximation (thick, dashed, red line), exact cross section times branching ratio into $\gamma \gamma$ (thin, black line), and exact cross section times branching ratio into $\gamma \gamma$ in the absence of mixing (dash-dotted, black line). 
magnitude than the product of stop-antistop coefficients, the Higgs coefficients dominate at minimal mixing. The stop-antistop width at $m_{H}=720 \mathrm{GeV}$ is now $\Gamma_{\tilde{t} \tilde{t}} \approx 0.44 \mathrm{GeV}$. This larger stop-antistop width has only a small effect on the cross section times branching ratio at minimal mixing, where the Higgs contribution is dominant, and changes the resonance widths only slightly at maximal mixing. Consequently, as in the case of $\kappa=8$ and $\Gamma_{\tilde{t}}=0.1 \mathrm{MeV}$, the cross section times branching ratio changes very little from minimal mixing to maximal mixing. This can be seen in the right panel of Fig. 4. In this case, the contributions from the two eigenstates sum to produce a total of the cross section times the branching ratio that deviates very little from the total of the cross section times the branching ratio in the absence of mixing. Again, the result from the narrow-resonance approximation for the cross section is essentially featureless, and it agrees well with the exact result.

\section{Differences between the Breit-Wigner resonance and the Coulomb-Schrödinger Green's function}

As we will see, many of the qualitative features of the model in which we replace the stop-antistop Green's function with a Breit-Wigner resonance persist when we model the stop-antistop Green's function with the CoulombSchrödinger Green's function. There are, however, several important differences.

First, the Coulomb-Schrödinger Green's function develops an imaginary part above the stop-antistop threshold, owing to the fact that the physical states can decay into a stop-antistop pair. In the case of large Higgs-stop-antistop coupling, this imaginary part can broaden the higher-mass physical state significantly and lead to a substantial reduction in its contribution to the cross section.

Second, the logarithm of $\tilde{E}$ in Eq. (34) can produce additional structure near the stop-antistop threshold. For the examples that we have considered, this additional structure appears to have a small effect on the cross section times the branching ratio to $\gamma \gamma$.

Third, the Coulomb-Schrödinger Green's function contains multiple bound-state poles. As we will see, the additional poles beyond the ground-state pole do not have a dramatic effect on the cross section times the branching ratio to $\gamma \gamma$.

Fourth, the Coulomb-Schrödinger Green's function has a much more complicated dependence on $\hat{s}$ than does the Breit-Wigner resonance. This situation is analyzed in detail in the Appendix. The generalization of the matrix in Eq. (42) is diagonalized by a similarity transformation that depends on $\hat{s}$. Each of the matrix eigenvalues $\alpha_{ \pm}$can have multiple poles in its inverse. The real parts of the poles locations, $m_{ \pm}$, and the imaginary parts of the pole locations, $m_{ \pm} \Gamma_{ \pm}$, determine the resonance widths, which are different than in the Breit-Wigner case. An important additional difference is that the residues of those poles, $Z_{ \pm}$, which are equal to unity in the Breit-Wigner case, can now have magnitudes that are larger or smaller than unity. This change in the pole residues is driven largely by the term that is proportional to $1 / \lambda$ in Eq. (34). However, the specific value of the residue depends on the other terms in Eq. (34), as well.

Fifth, in the Coulomb-Schrödinger analysis, we include the effects of the $t$-channel Higgs exchange on the stopantistop propagator. Had we included these effects in the Breit-Wigner analysis, they would have produced only small shifts in the mass of the Breit-Wigner resonance. In the Coulomb-Schrödinger analysis, it turns out that the $t$-channel Higgs exchange has a negligible effect for the case $\kappa=1$. However, it produces small, but noticeable, effects for the case $\kappa=8$, particularly for the smaller stop width. These $t$-channel-Higgs-exchange effects do not change the qualitative picture for the total cross section.

As we have mentioned, in the approximation in which a resonance amplitude is given by a Breit-Wigner form and the separation of the resonance from other resonances is small in comparison to the resonance widths, the contribution of a resonance to the cross section is proportional to the square of the maximum height of the absolute value of the amplitude times the full width at half maximum of the peak in the absolute value of the amplitude. Specifically, the cross-section contribution is proportional to $\left|Z_{ \pm}\right|^{2} / \Gamma_{ \pm}$.

\section{CASE OF THE COULOMB-SCHRÖDINGER GREEN'S FUNCTION}

In this section, we present numerical results for the $g g \rightarrow$ $\gamma \gamma$ amplitudes and the associated LHC cross sections at $\sqrt{s}=13 \mathrm{TeV}$ for the case in which the stop-antistop Green's function is calculated from the CoulombSchrödinger Green's function [Eq. (34)].

Heavy-Higgs production and decay rates depend not only on the Higgs coupling to the stop squark, but also on the Higgs couplings to the top and bottom quarks. As we have mentioned, we make use of an intermediate value of $\tan \beta$, setting $\tan \beta=\sqrt{m_{t} / m_{b}}$, where $m_{t}$ and $m_{b}$ are the $\overline{\mathrm{MS}}$ running masses at the scale $2 m_{\tilde{t}}$. At this value of $\tan \beta$, the Higgs decay width into third generation fermions is minimized and is about $1.2 \mathrm{GeV}$. The additional contributions to the Higgs production rate and decay width that are associated with Higgs couplings to stop-antistop pairs are automatically taken into account within our theoretical framework. The contributions to the total width from the decay of the heavy Higgs boson into pairs of Higgs bosons or heavy gauge bosons tend to be small, and we omit them in our analysis.

Our results depend strongly on the Higgs coupling to the stop-antistop pair and on the stop width. Therefore, we present numerical results for two representative cases of weak and strong Higgs coupling to the stop-antistop pair and for small and moderate values of the stop width. In 
particular, as in our analysis of the Breit-Wigner-resonance model, we give results for the cases $\kappa=1$ and $\Gamma_{\tilde{t}}=0.1 \mathrm{MeV}, \quad \kappa=1$ and $\Gamma_{\tilde{t}}=0.1 \mathrm{GeV}, \kappa=8$ and $\Gamma_{\tilde{t}}=0.1 \mathrm{MeV}$, and $\kappa=8$ and $\Gamma_{\tilde{t}}=0.1 \mathrm{GeV}$. We use the input values of the various parameters that were discussed in Sec. III B. We present results for various values of the Higgs mass and the partonic center-of-mass energy $\sqrt{\hat{s}}$.

In computing cross sections, we take into account only the gluon-gluon initiated process, which has been the focus of our discussion. As we have mentioned, the true stopantistop Green's function likely contains only a few bound states below threshold. Therefore, we give results that are obtained by taking into account only the first term or the first three terms in the sum in Eq. (34a). For comparison, we also give results that are based on the full CoulombSchrödinger Green's function in Eq. (34a). In the figures for the amplitudes below, we show, for clarity, the results that are obtained by retaining only one pole in the stop-antistop Green's function. That is, take only the $n=1$ term in the sum in Eq. (34a). In these figures, we show the following: (1) $\left|A_{\text {tot }}\right|$; (2) $\left|A_{H}^{\text {bare }}\right|=\left|A_{1}^{\text {bare }}+A_{2}^{\text {bare }}+A_{3}^{\text {bare }}+A_{4}^{\text {bare }}\right|$, where superscript "bare" means that the stop-antistop corrections to the Higgs propagator in Eq. (23), which are proportional to $C_{H \tilde{t} \tilde{t}}^{2}$, have been neglected; and (3) $\left|A_{\tilde{t} \tilde{t}}^{\text {bare }}\right|$, where $A_{\tilde{t} \tilde{t}}^{\text {bare }}=$ $A_{5}$ is the stop-antistop amplitude in the absence of Higgs coupling to the stop. Note that $A_{H}^{\text {bare }}$ contains all of the Higgsform-factor contributions that were discussed in Sec. II E. However, the absence of stop-antistop corrections to the Higgs propagator in $A_{H}^{\text {bare }}$ affects that amplitude in two important ways: (1) the Higgs-stop-antistop-mixing effects that lead to the displacement of the physical mass eigenvalues from threshold are not present, and (2) some of the corrections to the Higgs width that are associated with Higgs decays into stop-antistop pairs for Higgs masses above the stop-antistop threshold are not present.

We remind the reader that, as we have explained in Sec. III, the cross-section contribution of a resonance whose amplitude can be approximated by a Breit-Wigner form is proportional, in the narrow-width approximation, to the square of the maximum height of the absolute value of the amplitude times the full width at half maximum of the absolute value of the amplitude.

\section{A. $\kappa=1, \Gamma_{\tilde{t}}=0.1 \mathrm{MeV}$}

In this case, the Higgs boson couples only weakly to the stop, and the stop width is much less than the Higgs width.

In Fig. 6, we show $\left|A_{\text {tot }}\right|,\left|A_{H}^{\text {bare }}\right|$, and $\left|A_{\tilde{t} \tilde{t}}^{\text {bare }}\right|$ for $m_{H}=720,750$, and $780 \mathrm{GeV}$. The results for the three different Higgs masses show that, for $\kappa=1$, the mixing has a small effect on the physical masses, which remain close to their values in the absence of mixing. At $m_{H}=720 \mathrm{GeV}$ we can clearly identify the Higgs and stoponium contributions to the total amplitude. At $m_{H}=780 \mathrm{GeV}$, we can also see the separate contributions from the Higgs and the stoponium peaks, but we see a large increase in the width of the Higgs peak that is associated with the Higgs decay to a stop-antistop pair. At $m_{H}=750 \mathrm{GeV}$, where the mixing is maximal, the physical masses are slightly displaced relative to the Higgs and stoponium masses. Comparison of the upper left panel with the upper right panel shows that $\left|A_{\text {tot }}\right|$ for the Coulomb-Schrödinger Green's function and the Breit-Wigner Green's function are quite similar, although the lower-mass peak is somewhat broader and higher in the Coulomb-Schrödinger case. Note that, at maximal mixing, both physical peaks are much broader than the unmixed stoponium peak.

In Fig. 7, we show the total diphoton production cross section $\sigma_{\text {tot }}$ as a function of $m_{H}$. For comparison, we also show $\sigma_{H}^{\text {bare }}$ (which corresponds to $A_{H}^{\text {bare }}$ ) and $\sigma_{\tilde{t} \tilde{t}}^{\text {bare }}$ (which corresponds to $A_{\tilde{t} \tilde{t}}^{\text {bare }}$ ) as functions of $m_{H}$. Figure 7 shows that there are only small quantitative changes in $\sigma_{\text {tot }}$ as one includes additional stoponium poles in the stop-antistop Green's function.

A comparison of Fig. 7 with the right panel of Fig. 2 shows that the total cross section $\sigma_{\text {tot }}$ in the CoulombSchrödinger case has the same qualitative features as in the Breit-Wigner case. The Higgs cross section $\sigma_{H}^{\text {bare }}$ is much less than the stop-antistop cross section $\sigma_{\tilde{t} t}^{\text {bare }}$. The cross section is dominated by the width of the narrowest peak. At minimal mixing, this narrowest peak corresponds to the stoponium peak. At maximal mixing, the width of the narrowest physical peak is much greater than at minimal mixing, and the height shrinks roughly as the inverse of the width, resulting in a suppression of the cross section. As in the Breit-Wigner case (Sec. III B 1), this suppression is so great that it overwhelms the peaking effect that results from mixing of the short-distance coefficients. We see that, even at $m_{H}=720 \mathrm{GeV}$ and $m_{H}=780 \mathrm{GeV}$, mixing broadens the narrowest peak and reduces its height sufficiently that the total cross section is well below $\sigma_{\tilde{t} \tilde{t}}^{\text {bare }}$.

\section{B. $\kappa=1, \Gamma_{\tilde{t}}=0.1 \mathrm{GeV}$}

In this case, the Higgs boson still couples weakly to the stop, but the stop width is much closer to the Higgs width than in the previous example.

In Fig. 8, we show $\left|A_{\text {tot }}\right|,\left|A_{H}^{\text {bare }}\right|$, and $\left|A_{\tilde{t} \tilde{t}}^{\text {bare }}\right|$. The left and right panels show the results that are obtained in the Coulomb-Schrödinger case and the Breit-Wigner case, respectively. Again, these two choices for the stop-antistop Green's function result in qualitatively similar amplitudes. However, the height and width of the lower-mass resonance are both enhanced in the Coulomb-Schrödinger case relative to the Breit-Wigner case. At $m_{H}=750 \mathrm{GeV}$, where mixing is maximal, we see that the physical masses are displaced slightly relative to the Higgs and stoponium masses. Comparison with Fig. 6 shows that there is a marked increase in the width of the stoponium peak in 

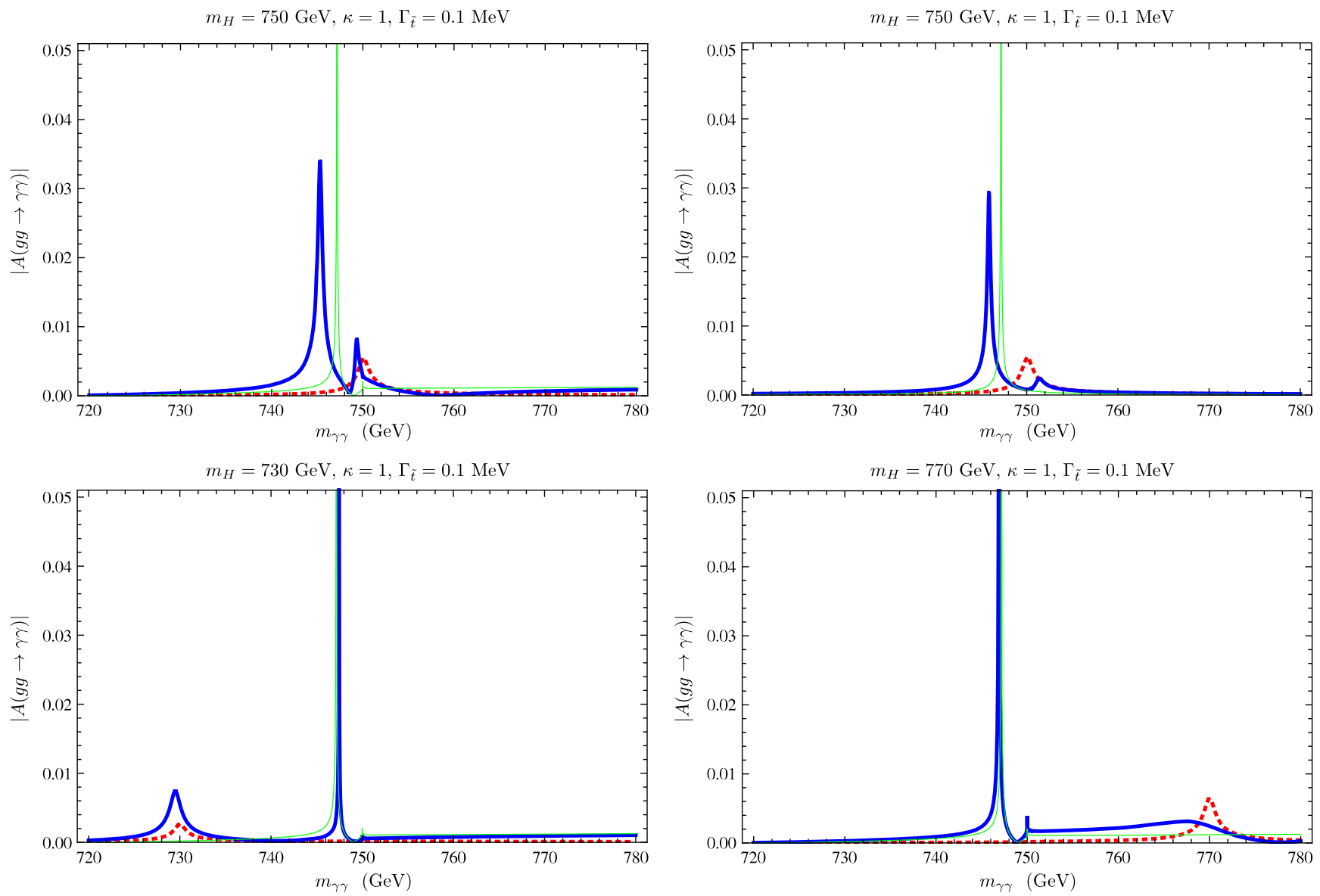

FIG. 6. Amplitudes for the case $\kappa=1, \Gamma_{\tilde{t}}=0.1 \mathrm{MeV}$. Upper left figure: the amplitudes $\left|A_{\text {tot }}\right|$ (thick, blue line), $\left|A_{\tilde{t} \tilde{t}}^{\text {bare }}\right|$ (thin, green line), and $\left|A_{H}^{\text {bare }}\right|$ (dashed, red line) vs $m_{\gamma \gamma}$ for $m_{H}=750 \mathrm{GeV}$. Upper right figure: the same amplitudes, but with the stop-antistop propagator replaced with a Breit-Wigner resonance, as in Eq. (39b). Lower left figure: the same as the upper left figure, but with $m_{H}=730 \mathrm{GeV}$. Lower right figure: the same as the upper left figure, but with $m_{H}=770 \mathrm{GeV}$.

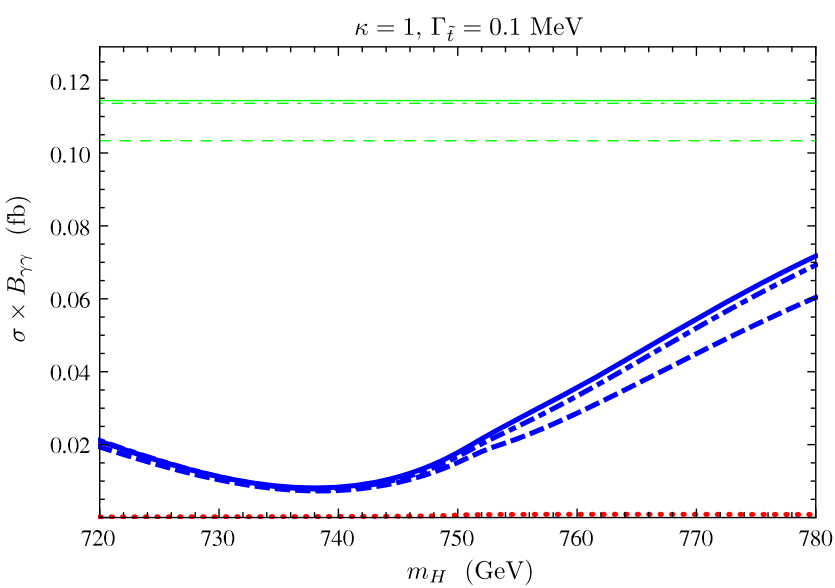

FIG. 7. Cross sections for the case $\kappa=1, \Gamma_{\tilde{t}}=0.1 \mathrm{MeV}: \sigma_{\text {tot }}$ (thick, blue lines), $\sigma_{H}^{\mathrm{bare}}$ (dotted, red line), and $\sigma_{\tilde{t} \tilde{t}}^{\mathrm{bare}}$ (thin, green lines) vs $m_{H}$. In the cases of $\sigma_{\text {tot }}$ and $\sigma_{\tilde{t} \tilde{t}}^{\text {bare }}$, the dashed, dasheddotted, and solid lines correspond, respectively, to taking 1, 3, or all terms in the sum in Eq. (34a).
$A_{\tilde{t} \tilde{t}}^{\text {bare }}$, owing to the increase in the stop width. Because of mixing effects, the width of the narrowest peak in $A_{\text {tot }}$ is much larger than the width of the stoponium peak in $A_{\tilde{t} \tilde{t}}^{\mathrm{bare}}$. The width of the narrowest peak in $A_{\text {tot }}$ is larger in Fig. 8 than in Fig. 6, owing to the increase in the width of the stop.

In Fig. 9, we display $\sigma_{\text {tot }}, \sigma_{H}^{\text {bare }}$, and $\sigma_{\tilde{t} \tilde{t}}^{\text {bare }}$, as functions of $m_{H}$. As can be seen, there are only small quantitative differences that are associated with the inclusion of additional stoponium poles in the stop-antistop Green's function.

A comparison of Fig. 9 with the right panel of Fig. 3 shows that the total cross section $\sigma_{\text {tot }}$ in the CoulombSchrödinger case again has the same qualitative features as in the Breit-Wigner case. However, there are minor differences in the shapes, and the Coulomb-Schrödinger cross section is considerably enhanced relative to the BreitWigner cross section, owing to the increase in the height and width of the lower-mass resonance, which can be seen in Fig. 8. Once again, the Higgs cross section $\sigma_{H}^{\text {bare }}$ is much less than the stop-antistop cross section $\sigma_{\tilde{t} \tilde{t}}^{\text {bare }}$, although $\sigma_{\tilde{t} \tilde{t}}^{\text {bare }}$ 

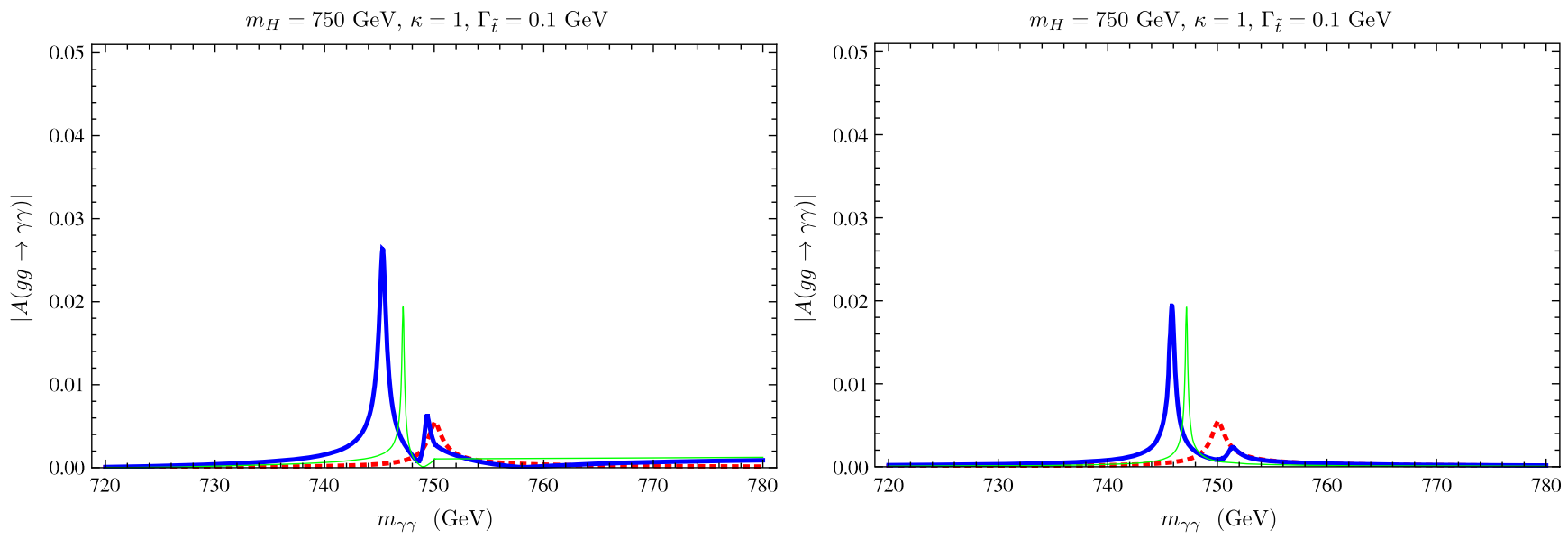

FIG. 8. Amplitudes for the case $\kappa=1, \Gamma_{\tilde{t}}=0.1 \mathrm{GeV}$. Left figure: the amplitudes $\left|A_{\text {tot }}\right|$ (thick, blue line), $\left|A_{\tilde{t} \tilde{t}}^{\text {bare }}\right|$ (thin, green line), and $\left|A_{H}^{\text {bare }}\right|$ (dashed, red line) vs $m_{\gamma \gamma}$ for $m_{H}=750 \mathrm{GeV}$. Right figure: the same amplitudes, but with the stop-antistop propagator replaced with a Breit-Wigner resonance, as in Eq. (39b).

is reduced in this broad-stop-width case in comparison to $\sigma_{\tilde{t} \tilde{t}}^{\text {bare }}$ in the small-stop-width case. Nevertheless, the cross section is dominated by the width of the narrowest peak, which corresponds, at minimal mixing, to the stoponium peak. We see that, in contrast with the small-stop-width case, the cross section now displays a peak at maximal mixing. As we explained in Sec. III B 2, this peak arises from the effects of mixing on the short-distance coefficients and is unrelated to threshold-enhancement effects. A peak persists in $\sigma_{\text {tot }}$ because, owing to the large stop width, the width of the narrowest peak in $A_{\text {tot }}$ does not change sufficiently between minimal and maximal mixing to reverse the peaking effect from the short-distance coefficients.$$
\text { C. } \kappa=8, \Gamma_{\tilde{t}}=0.1 \mathrm{MeV}
$$

Next, we discuss the case of a large Higgs-stop-antistop coupling, $\kappa=8$, and a small stop width, $\Gamma_{\tilde{t}}=0.1 \mathrm{MeV}$.

Large values of the Higgs-stop-antistop coupling have a very large impact on the diphoton production rate, as has been emphasized in the context of perturbative calculations in Ref. [15]. As we will see, for $\kappa=8$, the Higgs-stopantistop mixing effects become dramatic, and it is essential to include those effects, which go beyond the effects that are contained in fixed-order perturbation theory, in order to compute the diphoton rate reliably.

In Fig. 10 , we show $\left|A_{\text {tot }}\right|,\left|A_{H}^{\text {bare }}\right|$, and $\left|A_{\tilde{t} t}^{\text {bare }}\right|$ for $m_{H}=750 \mathrm{GeV}$. The left and right panels show the results that are obtained in the Coulomb-Schrödinger case and the Breit-Wigner case, respectively. In both the CoulombSchrödinger case and the Breit-Wigner case, there is a clear shift of the physical poles at maximal mixing away from the stop-antistop threshold. However, there are several important differences between the Coulomb-Schrödinger amplitude and the Breit-Wigner amplitude. First, at maximal mixing, the larger-mass peak that is present in the
Breit-Wigner amplitude has almost disappeared in the Coulomb-Schrödinger amplitude. The reason for this is that the larger-mass physical state has a very large decay width into a stop-antistop pair. The very narrow peak in the Coulomb-Schrödinger amplitude near threshold does not correspond to the larger-mass peak in the Breit-Wigner amplitude. Rather, it arises from the logarithmic term in Eq. (34). It gives a small contribution to the cross section. We also see that the width of the lower-mass peak is significantly smaller in the Coulomb-Schrödinger amplitude than in the Breit-Wigner amplitude, while its height is about the same. This results in a reduced contribution of this peak to the cross section.

In Fig. 11 , we show $\sigma_{\text {tot }}, \sigma_{H}^{\text {bare }}$, and $\sigma_{\tilde{t} \tilde{t}}^{\text {bare }}$, as functions of $m_{H}$. A comparison with the right panel of Fig. 4 shows that the shape of $\sigma_{\text {tot }}$ in the Coulomb-Schrödinger case is similar

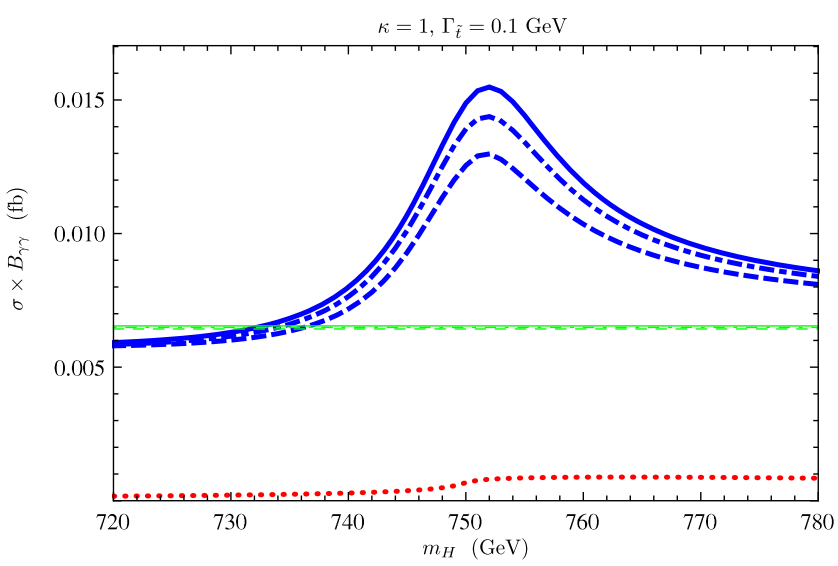

FIG. 9. Cross sections for the case $\kappa=1, \Gamma_{\tilde{t}}=0.1 \mathrm{GeV}: \sigma_{\text {tot }}$ (thick, blue lines), $\sigma_{H}^{\text {bare }}$ (dotted, red line), and $\sigma_{\tilde{t} \tilde{t}}^{\text {bare }}$ (thin, green lines) vs $m_{H}$. In the cases of $\sigma_{\text {tot }}$ and $\sigma_{\tilde{t} \tilde{t}}^{\text {bare }}$, the dashed, dasheddotted, and solid lines correspond, respectively, to taking 1, 3, or all terms in the sum in Eq. (34a). 

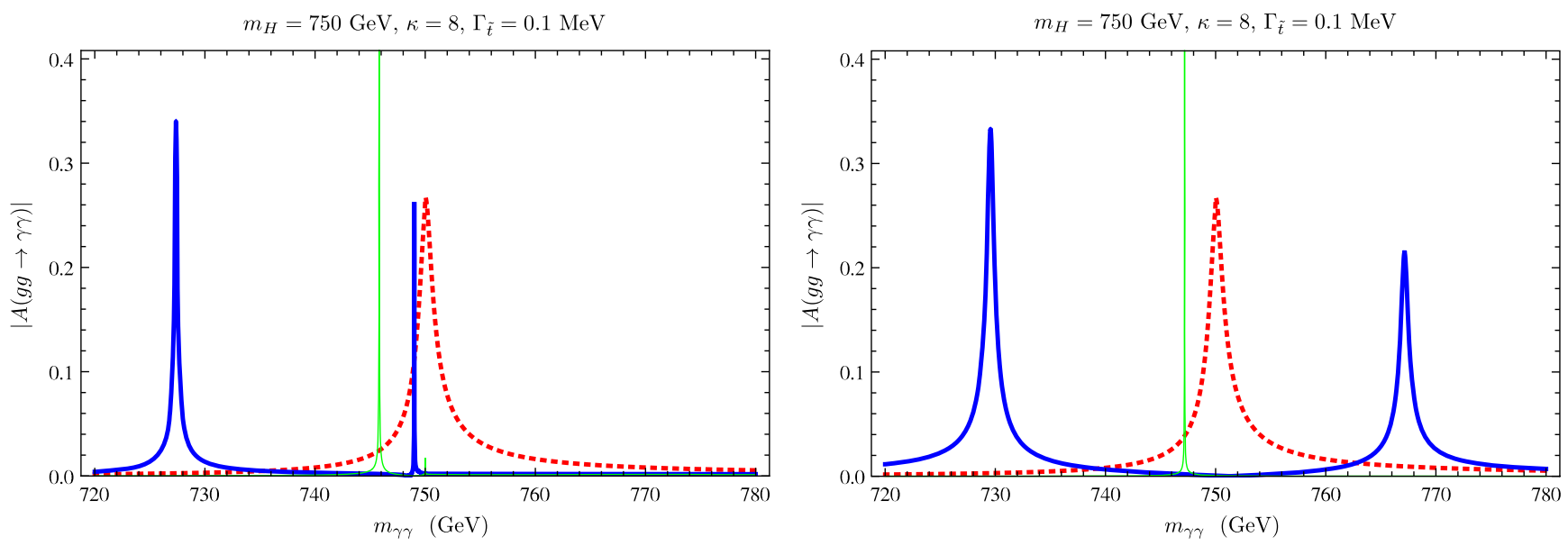

FIG. 10. Amplitudes for the case $\kappa=8, \Gamma_{\tilde{t}}=0.1 \mathrm{MeV}$. Left figure: the amplitudes $\left|A_{\text {tot }}\right|$ (thick, blue line), $\left|A_{\tilde{t} \tilde{t}}^{\text {bare }}\right|$ (thin, green line), and $\left|A_{H}^{\text {bare }}\right|$ (dashed, red line) vs $m_{\gamma \gamma}$ for $m_{H}=750 \mathrm{GeV}$. Right figure: the same amplitudes, but with the stop-antistop propagator replaced with a Breit-Wigner resonance, as in Eq. (39b).

to the shape of $\sigma_{\text {tot }}$ in the Breit-Wigner case: Both are fairly featureless, although the slopes are different. As in the case of the Breit-Wigner cross section, this featureless nature of the Coulomb-Schrödinger cross section is a consequence of three properties of the amplitude: (1) the short-distance coefficients are dominated by their imaginary parts, and so the mixing of the short-distance coefficients produces no pronounced peaks or dips; (2) the Higgs short-distance coefficients dominate over the stop-antistop short-distance coefficients, and so the stoponium peak does not contribute greatly to the cross section; and (3) the height and width of the lower-mass resonance are not very different from those of a Higgs resonance over the range of $m_{H}$.

The cross section is considerably smaller in the Coulomb-Schrödinger case than in the Breit-Wigner case.

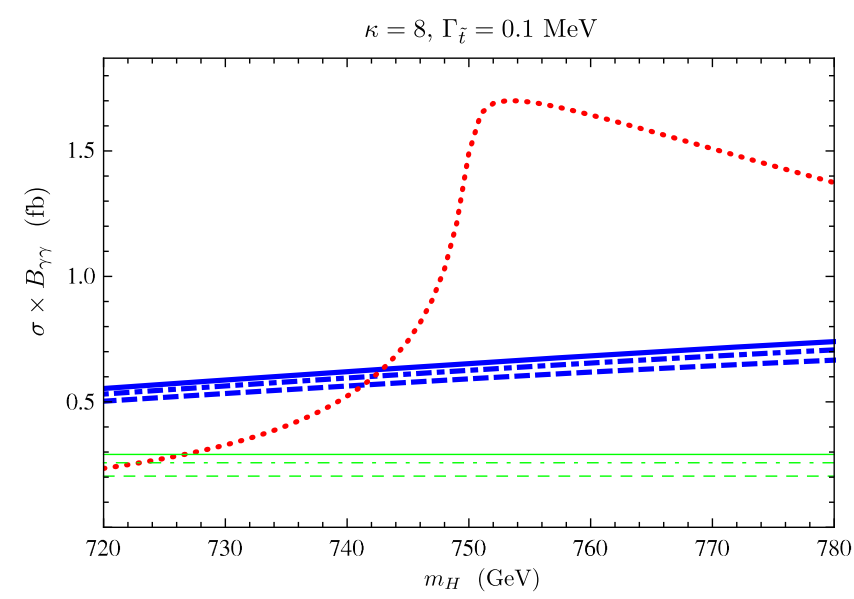

FIG. 11. Cross sections for the case $\kappa=8, \Gamma_{\tilde{t}}=0.1 \mathrm{MeV}: \sigma_{\text {tot }}$ (thick, blue lines), $\sigma_{H}^{\text {bare }}$ (dotted, red line), and $\sigma_{\tilde{t} \tilde{t}}^{\text {bare }}$ (thin, green lines) vs $m_{H}$. In the cases of $\sigma_{\text {tot }}$ and $\sigma_{\tilde{t} \tilde{t}}^{\text {bare }}$, the dashed, dasheddotted, and solid lines correspond, respectively, to taking 1, 3, or all terms in the sum in Eq. (34a).
The reasons for this are the disappearance of the highermass peak in the Coulomb-Schrödinger case, owing to its large width into a stop-antistop pair, and the narrowing of the lower-mass peak while its width remains constant.

In the case $\kappa=8$, in comparison to the case of $\kappa=1, \sigma_{H}^{\text {bare }}$ is greatly enhanced by the large Higgs-stop-antistop coupling and is greater than $\sigma_{\tilde{t} \tilde{t}}^{\text {bare }}$. At $m_{H}=750 \mathrm{GeV}$, we see the threshold enhancement of $\sigma_{H}^{\text {bare }}$ that is associated with the Higgs diphoton and digluon form factors. However, $\sigma_{\text {tot }}$ does not show a similar threshold enhancement because of the shifts of the masses of the physical states away from threshold. Although $\sigma_{\text {tot }}$ is enhanced at small values of the Higgs mass with respect to $\sigma_{H}^{\text {bare }}$, this enhancement becomes a suppression of factors of a few at values of the Higgs mass that are close to the stop-antistop production threshold. ${ }^{12}$

\section{D. $\kappa=8, \Gamma_{\tilde{t}}=0.1 \mathrm{GeV}$}

Finally, we discuss the case of a large Higgs-stop-antistop coupling, $\kappa=8$, and a large stop width, $\Gamma_{\tilde{t}}=0.1 \mathrm{GeV}$.

In Fig. 12, we show $\left|A_{\text {tot }}\right|,\left|A_{H}^{\text {bare }}\right|$, and $\left|A_{\tilde{t} \tilde{t}}^{\text {bare }}\right|$ for $m_{H}=750 \mathrm{GeV}$. The left and right panels show the results that are obtained in the Coulomb-Schrödinger case and the Breit-Wigner case, respectively. As in the case of the smaller stop width, at maximal mixing there is a clear shift of the physical poles away from the stop-antistop threshold. There are again several important differences between the Coulomb-Schrödinger amplitude and the Breit-Wigner amplitude. At maximal mixing, the largermass peak that is present in the Breit-Wigner amplitude has almost disappeared in the Coulomb-Schrödinger amplitude,

\footnotetext{
${ }^{12} \mathrm{We}$ have also examined the cross sections for the intermediate value $\kappa=5$. The results are qualitatively similar to those at $\kappa=8$, except that $\sigma_{H}^{\text {bare }}$ is reduced relative to $\sigma_{\text {tot }}$, and, so, there is a mild enhancement of $\sigma_{\text {tot }}$ relative to $\sigma_{H}^{\text {bare }}$.
} 

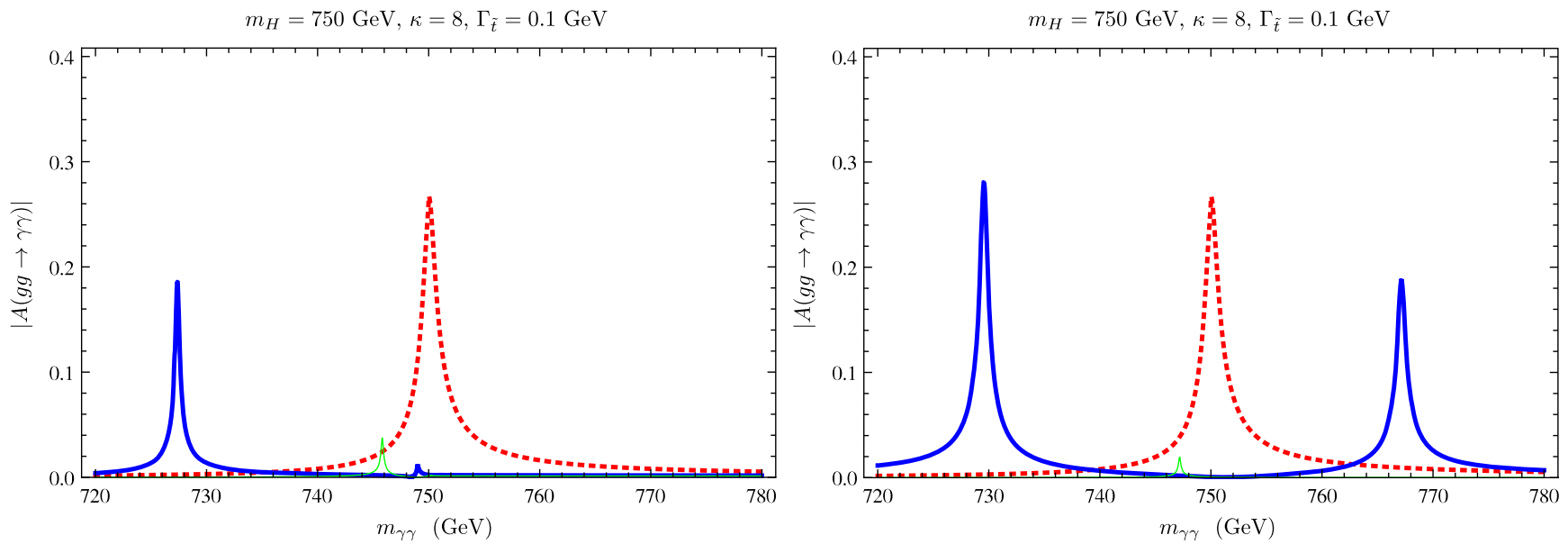

FIG. 12. Amplitudes for the case $\kappa=8, \Gamma_{\tilde{t}}=0.1 \mathrm{GeV}$. Left figure: the amplitudes $\left|A_{\text {tot }}\right|$ (thick, blue line), $\left|A \tilde{t} \tilde{t}^{\text {bare }}\right|$ (thin, green line), and $\left|A_{H}^{\text {bare }}\right|$ (dashed, red line) vs $m_{\gamma \gamma}$ for $m_{H}=750 \mathrm{GeV}$. Right figure: the same amplitudes, but with the stop-antistop propagator replaced with a Breit-Wigner resonance, as in Eq. (39b).

owing to the large decay width of the larger-mass physical state into a stop-antistop pair. There is again a structure near threshold that appears only in the Coulomb-Schrödinger amplitude that arises from the logarithmic term in Eq. (34), but in this large-stop-width case, the structure has nearly disappeared. We also see that both the height and the width of the lower-mass peak are significantly smaller in the Coulomb-Schrödinger amplitude than in the Breit-Wigner amplitude. These changes in the lower-mass peak result in a greatly reduced contribution of the lower-mass peak to the cross section. In this larger-stop-width case, the stoponium peak in $\left|A_{H}^{\text {bare }}\right|$ is much broader than in the smaller-stopwidth case and is so small as to be nearly invisible.

In Fig. 13, we show $\sigma_{\text {tot }}, \sigma_{H}^{\text {bare }}$, and $\sigma_{\tilde{t} \tilde{t}}^{\text {bare }}$, as functions of $m_{H}$. A comparison with the right panel of Fig. 5 shows that

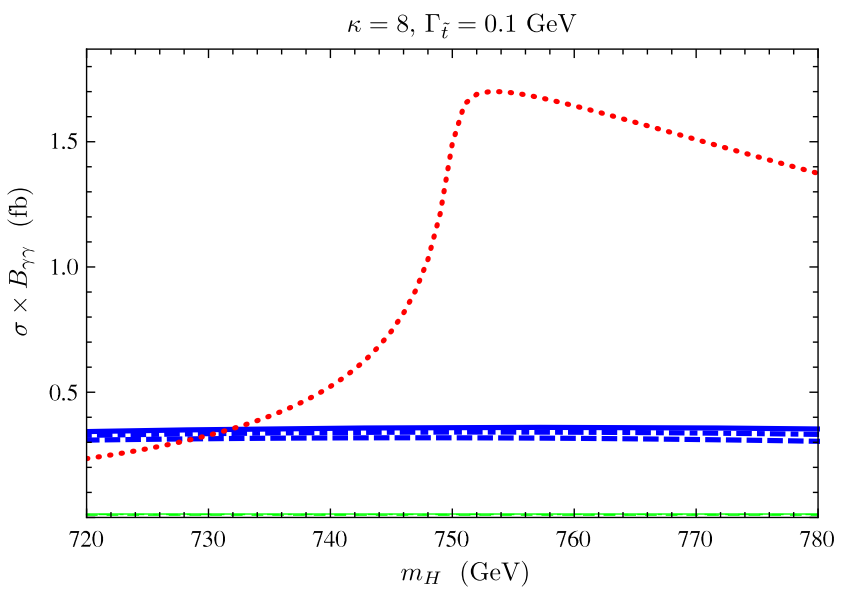

FIG. 13. Cross sections for the case $\kappa=8, \Gamma_{\tilde{t}}=0.1 \mathrm{GeV}: \sigma_{\text {tot }}$ (thick, blue lines), $\sigma_{H}^{\text {bare }}$ (dotted, red line), and $\sigma_{\tilde{t} \tilde{t}}^{\text {bare }}$ (thin, green lines) vs $m_{H}$. In the cases of $\sigma_{\text {tot }}$ and $\sigma_{\tilde{t} \tilde{t}}^{\text {bare }}$, the dashed, dasheddotted, and solid lines correspond, respectively, to taking 1, 3, or all terms in the sum in Eq. (34a). the shape of $\sigma_{\text {tot }}$ in the Coulomb-Schrödinger case is again similar to the shape of $\sigma_{\text {tot }}$ in the Breit-Wigner case. The cross section is featureless for the reasons that we mentioned in the discussion of the cross section for the narrower stop width. For this larger stop-width, the cross section is much smaller in the Coulomb-Schrödinger case than in the Breit-Wigner case. As in the case of the smaller stop width, the higher-mass peak has disappeared in the CoulombSchrödinger amplitude, owing to the large width of the higher-mass peak into a stop-antistop pair. Furthermore, the reduction in both the height and the width of the lowermass peak has resulted in an additional reduction of $\sigma_{\text {tot }}$, relative to its values in the smaller-stop-width case.

Again, we see that the larger value of the Higgs-stopantistop coupling greatly enhances $\sigma_{H}^{\text {bare }}$. Owing to the larger stop width, $\sigma_{\tilde{t} \tilde{t}}^{\text {bare }}$ has almost disappeared in the figure. At $m_{H}=750 \mathrm{GeV}$, we again see the threshold enhancement of $\sigma_{H}^{\text {bare }}$ that is associated with the Higgs diphoton and digluon form factors. As in the smaller-stop-width-case, $\sigma_{\text {tot }}$ does not show a similar threshold enhancement because of the shifts of the masses of the physical states away from threshold. In this larger-stop-width case, $\sigma_{\text {tot }}$ is comparable to $\sigma_{H}^{\text {bare }}$ at small values of the Higgs mass and is much smaller than $\sigma_{H}^{\text {bare }}$ at values of the Higgs mass that are close to the stop-antistop production threshold. ${ }^{13}$

\section{CONCLUSIONS}

The system of a heavy Higgs boson that is coupled to a stop-antistop pair exhibits some interesting field-theoretic phenomena near the stop-antistop production threshold.

\footnotetext{
${ }^{13}$ At $\kappa=5$, the cross-section results are qualitatively similar to those at $\kappa=8$, except that $\sigma_{H}^{\text {bare }}$ is reduced relative to $\sigma_{\text {tot }}$, and, so, there is a mild enhancement of $\sigma_{\text {tot }}$ relative to $\sigma_{H}^{\text {bare }}$, except in a small region of $m_{H}$ between 750 and $770 \mathrm{GeV}$.
} 
This system has attracted interest in the context of the production of a heavy Higgs boson near the stop-antistop threshold and its subsequent decay to two photons [15], owing to the perturbative enhancement of the Higgs couplings to photons and gluons near threshold. However, as is well known, the appearance of Coulomb infrared singularities near threshold invalidates the use of fixed-order perturbation theory. These Coulomb singularities first appear at two-loop order in the Higgs-to-diphoton and Higgs-to-digluon form factors. They occur when $v$ (onehalf the stop-antistop relative velocity in the stop-antistop CM frame) goes to zero, and they are a manifestation of the general phenomenon of $1 / v$ enhancements of two-particle amplitudes near threshold. These enhancements require an all-orders treatment, and they lead, among other things, to the formation of stoponium bound states.

A discussion of nonperturbative threshold effects is given in Ref. [15] and focuses on the enhancements of the Higgsto-diphoton and Higgs-to-digluon form factors that are induced by the stop-antistop bound states [16]. The discussion in Ref. [15] suggests that nonperturbative threshold effects produce an enhancement of the digluon-to-diphoton cross section, relative to the predictions for the cross section that are based on perturbative treatments of the Higgs-todiphoton and Higgs-to-digluon form factors near threshold. However, a correct treatment of the threshold effects also requires a complete analysis of Higgs-stop-antistop mixing effects.

In this paper, we have formulated the calculation of the threshold enhancements to the digluon-to-diphoton cross section in terms of the scalar-quark analogues of the effective field theories NRQED and NRQCD. Our treatment is valid up to corrections of relative order $v^{2}$. The effective theory gives a complete accounting of Higgs-stopantistop mixing in the threshold region. We have studied these enhancement and mixing effects numerically by making use of a model Green's function for the stopantistop system, namely, the Coulomb-Schrödinger Green's function. The Coulomb-Schrödinger Green's function does not correctly account for the QCD confining potential, which should be Coulombic only for the lowest-lying stoponium bound states. Therefore, we have considered the case in which the expression for the CoulombSchrödinger Green's function is truncated so that it contains only a few bound states. This approach retains only bound states for which the Coulombic approximation is expected to be valid, and it is in keeping with the actual stoponium spectrum, which is expected have only a few bound states. At a qualitative level, we have checked that the results that we have obtained are independent of the number of bound states that we have retained. Moreover, the quantitative differences that are associated with the inclusion of heavier bounds states are small, giving us confidence that our conclusions are not dependent on the specifics of the model Green's function that we have chosen.
We have also investigated a simplified model in which the stop-antistop Green's function is represented by a simple Breit-Wigner resonance. This simplified model exhibits some, but not all, of the qualitative features of the more complicated Coulomb-Schrödinger model.

We have found that the Higgs-stop-antistop mixing produces three general effects that are very significant. First, the mixing leads to mass eigenstates whose widths are larger than the widths of the stoponium states. For a single stoponium state and for large values of the Higgsstop-antistop coupling, the widths of the mass eigenstates at threshold approach the average of the Higgs and stoponium widths. These increases in the widths, and the concomitant reductions in the peak heights, reduce the contributions to the cross section relative to the contribution that would be obtained from a narrow stoponium state. Second, the physical masses are shifted from the input Higgs and stoponium masses, and, when the Higgs mass is near threshold, the physical masses are displaced away from the threshold region. This effect is particularly important for large values of the Higgs-stop-antistop coupling and can render the perturbative threshold enhancements inoperative. Third, when the Higgs-stop-antistop coupling is large, the displacement of the mass of the higher-mass physical state to a point above threshold can give that state a very large width into a stop-antistop pair, resulting in a drastic reduction of its contribution to the cross section.

In addition, to these general effects, there are some effects that depend on the details of the couplings of the Higgs boson and the stop-antistop pair to photons and gluons and on the details of the Coulomb-Schrödinger Green's function. For example, the couplings can mix in such a way as to produce a peak near threshold that has nothing to do with the threshold enhancements that are associated with the perturbative Higgs-digluon and Higgs-diphoton form factors. The Coulomb-Schrödinger Green's function can also lead to changes in the heights and widths of the physical peaks in the amplitudes, relative to their heights and widths in the simple Breit-Wigner model. These effects are driven largely by the term of lowest order in $\alpha_{s}$, in the Coulomb-Schrödinger Green's function. That term is universal in that it is independent of the nature of the squark-antiquark static potential. However, the details of the effects that arise from it seem to depend on nonuniversal features of the CoulombSchrödinger Green's function.

In general, for large values of the heavy-Higgs coupling to the stop-antistop pair, the mixing effects result in suppressions of the digluon-to-diphoton cross section at threshold relative to the cross section that is predicted in one-loop perturbation theory. The precise suppression factor depends not only on the Higgs-stop-antistop coupling but also on the stop width. We remind the reader that, because our focus is on the formulation of the calculation and on the qualitative features of the threshold physics, we have computed the Higgs couplings to digluons and diphotons at the one-loop 
level, and, so, one should take care in comparing our numerical results with those in the literature, which often include two-loop effects.

Although we have concentrated on the case of the Higgsstop-antistop interaction, the theoretical framework that we have developed is applicable to the coupling of Higgs bosons to other scalar particles in the region near the particle-antiparticle threshold. It can also be generalized easily to the case of a Higgs boson coupled to heavy fermions and to calculations of rates to different final states. For example, one could study the case of a $\tau^{+} \tau^{-}$final state by replacing the $\gamma \gamma$ short-distance coefficients in Eq. (31a) with the corresponding $\tau^{+} \tau^{-}$short-distance coefficients. ${ }^{14}$ We reserve the study of these additional cases for a separate publication.

\section{ACKNOWLEDGMENTS}

G. T. B. and H. S. C. would like to thank Estia Eichten for a helpful discussion. C. E. M. W. would like to thank Marcela Carena, Abdelhak Djouadi, Ahmed Ismail, Ian Low, Steve Martin, and Nausheen Shah for useful discussions. The work of G. T. B., H. S. C., and C. E. M. W. is supported by the U.S. Department of Energy, Division of
High Energy Physics, under Contract No. DE-AC0206CH11357. The submitted manuscript has been created in part by UChicago Argonne, LLC, Operator of Argonne National Laboratory. Argonne, a U.S. Department of Energy Office of Science laboratory, is operated under Contract No. DE-AC02-06CH11357. The U.S. Government retains for itself, and others acting on its behalf, a paid-up nonexclusive, irrevocable worldwide license in said article to reproduce, prepare derivative works, distribute copies to the public, and perform publicly and display publicly, by or on behalf of the Government. The work of C.E. M. W. at the University of Chicago is partially supported by the U.S. Department of Energy, under Contract No. DE-SC0009924. The work of H. S. C. at CERN is partially supported by the Korean Research Foundation through the CERN-Korea fellowship program.

\section{APPENDIX: DIAGONAL FORM OF THE AMPLITUDE IN THE GENERAL CASE}

In the general case, which includes the example of the Coulomb-Schrödinger Green's function, we can write Eq. (31a) as

$$
A_{\mathrm{tot}}(g g \rightarrow \gamma \gamma)=\left(\begin{array}{ll}
C_{g g H} & \hat{C}_{g \tilde{t} \tilde{t}}
\end{array}\right)\left(\begin{array}{cc}
S_{H}^{-1}(\hat{s}) & -\hat{C}_{H \tilde{t} \tilde{t}} \\
-\hat{C}_{H \tilde{t} \tilde{t}} & \hat{G}_{\tilde{t} \tilde{t}}^{-1}(\hat{s})
\end{array}\right)^{-1}\left(\begin{array}{c}
C_{\gamma \gamma H} \\
\hat{C}_{\gamma \gamma \tilde{t} \tilde{t}}
\end{array}\right),
$$

where $\hat{G}_{\tilde{t} \tilde{t}}(\hat{s})=\tilde{G}_{\tilde{t} \tilde{t}}(\hat{s}) / N_{\tilde{t} \tilde{t}}^{2}$, with $N_{\tilde{t} \tilde{t}}^{2}$ given in Eq. (40). ${ }^{15}$ The eigenvalues of the matrix in Eq. (A1) whose inverse is taken are given by

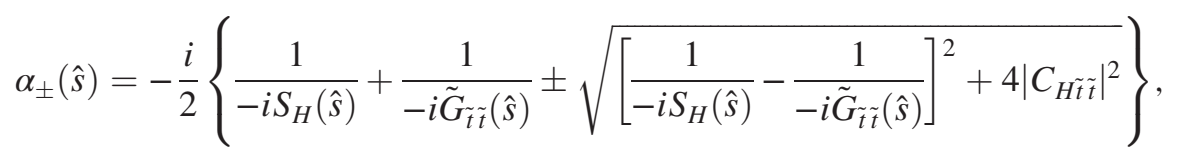

and the tangent of the rotation angle of the similarity transformation that diagonalizes that matrix is given by

$$
\tan [\theta(\hat{s})]=\frac{2\left|\hat{C}_{H \tilde{t} \tilde{t}}\right|}{\sqrt{\left[\frac{1}{-i S_{H}(\hat{s})}-\frac{1}{-i \tilde{G}_{\tilde{t} \tilde{t}}(\hat{s})}\right]^{2}+4\left|\hat{C}_{H \tilde{t} \tilde{t}}\right|^{2}}+\left[\frac{1}{-i S_{H}(\hat{s})}-\frac{1}{-i \tilde{G}_{\tilde{t} \tilde{t}(\hat{s})}}\right]} .
$$

We see that both the eigenvalues and the rotation angle now depend on $\hat{s}$.

The physical-state poles are located at the values $\hat{s}=\hat{s}_{ \pm}$ for which $\alpha_{ \pm}(\hat{s})$ vanishes. [Note that there may be more

\footnotetext{
${ }^{14}$ We note that $C_{\tau^{+} \tau^{-} \tilde{t} \tilde{t}}$ vanishes if one neglects electromagnetic and weak interactions.

${ }^{15}$ The choice of $N_{\tilde{t} \tilde{t}}^{2}$ is somewhat arbitrary. Here, we have chosen $N_{\tilde{t} \tilde{t}}^{2}$ so as to be consistent with the choice that we made in the Breit-Wigner case.
}

than one value of $\hat{s}_{ \pm}$for which $\alpha_{ \pm}(\hat{s})$ vanishes.] Near a pole, the eigenvalues of the inverse matrix that appears in Eq. (A1) are

$$
\frac{i}{Z_{ \pm}^{-1}\left(\hat{s}-m_{ \pm}^{2}\right)+i I_{ \pm}}=\frac{i Z_{ \pm}}{\hat{s}-m_{ \pm}^{2}+i m_{ \pm} \Gamma_{ \pm}},
$$

where

$$
m_{ \pm}^{2}=\operatorname{Re}\left(\hat{s}_{ \pm}\right),
$$




$$
\begin{gathered}
I_{ \pm}=\left.\operatorname{Im}\left[i \alpha_{ \pm}(\hat{s})\right]\right|_{\hat{s}=m_{ \pm}^{2}}, \\
Z_{ \pm}^{-1}=\left.\frac{\partial}{\partial \hat{s}} \operatorname{Re}\left[i \alpha_{ \pm}(\hat{s})\right]\right|_{\hat{s}=m_{ \pm}^{2}},
\end{gathered}
$$

$$
m_{ \pm} \Gamma_{ \pm}=I_{ \pm} Z_{ \pm}
$$

and the tangent of the rotation angle is given by

$$
\tan \left[\theta\left(m_{ \pm}^{2}\right)\right]=\left.\tan [\theta(\hat{s})]\right|_{\hat{s}=m_{ \pm}} .
$$

[1] H. E. Haber and G. L. Kane, Phys. Rep. 117, 75 (1985).

[2] H. P. Nilles, Phys. Rep. 110, 1 (1984).

[3] M. Drees, R. Godbole, and P. Roy, Theory and Phenomenology of Sparticles (World Scientific, Singapore, 2004).

[4] H. Baer and X. Tata, Weak Scale Supersymmetry: From Superfields to Scattering Events (Cambridge University Press, Cambridge, England, 2006).

[5] S. P. Martin, Adv. Ser. Dir. High Energy Phys. 21, 1 (2010); 18, 1 (1998).

[6] M. Drees and M. M. Nojiri, Phys. Rev. D 49, 4595 (1994).

[7] S. P. Martin, Phys. Rev. D 77, 075002 (2008).

[8] S. P. Martin and J. E. Younkin, Phys. Rev. D 80, 035026 (2009).

[9] J. E. Younkin and S. P. Martin, Phys. Rev. D 81, 055006 (2010).

[10] Y. Kats and M. J. Strassler, J. High Energy Phys. 05 (2016) 092.

[11] D. Choudhury and K. Ghosh, arXiv:1605.00013.

[12] M. Carena, P. Huang, A. Ismail, I. Low, N. R. Shah, and C. E. M. Wagner, Phys. Rev. D 94, 115001 (2016).

[13] G. C. Branco, P. M. Ferreira, L. Lavoura, M. N. Rebelo, M. Sher, and J. P. Silva, Phys. Rep. 516, 1 (2012).

[14] M. Drees and K. I. Hikasa, Phys. Rev. D 41, 1547 (1990).

[15] A. Djouadi and A. Pilaftsis, Phys. Lett. B 765, 175 (2017).

[16] K. Melnikov, M. Spira, and O. I. Yakovlev, Z. Phys. C 64, 401 (1994).

[17] W. E. Caswell and G. P. Lepage, Phys. Lett. 167B, 437 (1986).

[18] G. P. Lepage, L. Magnea, C. Nakhleh, U. Magnea, and K. Hornbostel, Phys. Rev. D 46, 4052 (1992).

[19] G. T. Bodwin, E. Braaten, and G. P. Lepage, Phys. Rev. D 51, 1125 (1995); 55, 5853(E) (1997).

[20] ATLAS Collaboration, Report No. ATLAS-CONF-2016029.

[21] CMS Collaboration, Report No. CMS-PAS-EXO-16-018.

[22] A. Strumia, arXiv:1605.09401.

[23] J. A. Casas, A. Lleyda, and C. Munoz, Nucl. Phys. B471, 3 (1996).

[24] N. Blinov and D. E. Morrissey, J. High Energy Phys. 03 (2014) 106.

[25] D. Chowdhury, R. M. Godbole, K. A. Mohan, and S. K. Vempati, J. High Energy Phys. 02 (2014) 110.

[26] C. Kim, A. Idilbi, T. Mehen, and Y. W. Yoon, Phys. Rev. D 89, 075010 (2014).

[27] C. W. Bauer, S. Fleming, D. Pirjol, and I. W. Stewart, Phys. Rev. D 63, 114020 (2001).
[28] M. Spira, A. Djouadi, D. Graudenz, and P. M. Zerwas, Nucl. Phys. B453, 17 (1995).

[29] R. V. Harlander, S. Liebler, and H. Mantler, Comput. Phys. Commun. 184, 1605 (2013).

[30] E. Bagnaschi, R. V. Harlander, S. Liebler, H. Mantler, P. Slavich, and A. Vicini, J. High Energy Phys. 06 (2014) 167.

[31] S. Dittmaier, P. Häfliger, M. Krämer, M. Spira, and M. Walser, Phys. Rev. D 90, 035010 (2014).

[32] F. Wilczek, Phys. Rev. Lett. 39, 1304 (1977).

[33] J. R. Ellis, M. K. Gaillard, D. V. Nanopoulos, and C. T. Sachrajda, Phys. Lett. 83B, 339 (1979).

[34] J. F. Gunion, H. E. Haber, G. L. Kane, and S. Dawson, Front. Phys. 80, 1 (2000).

[35] J. R. Ellis, M. K. Gaillard, and D. V. Nanopoulos, Nucl. Phys. B106, 292 (1976).

[36] M. A. Shifman, A. I. Vainshtein, M. B. Voloshin, and V. I. Zakharov, Yad. Fiz. 30, 1368 (1979) [Sov. J. Nucl. Phys. 30, 711 (1979)].

[37] A. Djouadi, M. Spira, and P. M. Zerwas, Phys. Lett. B 311, 255 (1993).

[38] N. Brambilla, A. Pineda, J. Soto, and A. Vairo, Nucl. Phys. B566, 275 (2000).

[39] H. K. Dreiner, M. E. Krauss, B. O’Leary, T. Opferkuch, and F. Staub, Phys. Rev. D 94, 055013 (2016).

[40] E. Eichten, K. Gottfried, T. Kinoshita, J. B. Kogut, K. D. Lane, and T. M. Yan, Phys. Rev. Lett. 34, 369 (1975); 36, 1276(E) (1976).

[41] K. Melnikov and A. Yelkhovsky, Nucl. Phys. B528, 59 (1998).

[42] Y. Kiyo, A. Pineda, and A. Signer, Nucl. Phys. B841, 231 (2010).

[43] G. S. Bali, B. Bolder, N. Eicker, T. Lippert, B. Orth, P. Ueberholz, K. Schilling, and T. Struckmann (SESAM and TXL Collaborations), Phys. Rev. D 62, 054503 (2000).

[44] S. Kim, Phys. Rev. D 92, 094505 (2015).

[45] K. Hagiwara, K. Kato, A. D. Martin, and C. K. Ng, Nucl. Phys. B344, 1 (1990).

[46] M. Baumgart and A. Katz, J. High Energy Phys. 08 (2012) 133.

[47] J. Pumplin, D. R. Stump, J. Huston, H. L. Lai, P. M. Nadolsky, and W. K. Tung, J. High Energy Phys. 07 (2002) 012.

[48] B. C. Allanach, P. S. B. Dev, and K. Sakurai, Phys. Rev. D 93, 035010 (2016).

[49] E. Braaten and J. P. Leveille, Phys. Rev. D 22, 715 (1980).

[50] M. Drees and K. I. Hikasa, Phys. Lett. B 240, 455 (1990); 262, 497(E) (1991). 